\title{
Tropical Cyclone Kinematic Structure Retrieved from Single-Doppler Radar Observations. Part I: Interpretation of Doppler Velocity Patterns and the GBVTD Technique
}

\author{
Wen-Chau LeE \\ National Center for Atmospheric Research, * Boulder, Colorado \\ Ben Jong-Dao Jou, Pao-Liang Chang, ${ }^{+}$And Shiung-Ming DenG ${ }^{\#}$ \\ Department of Atmospheric Sciences, National Taiwan University, Taipei, Taiwan
}

(Manuscript received 22 April 1998, in final form 11 September 1998)

\begin{abstract}
Deducing the three-dimensional primary circulation of landfalling tropical cyclones (TCs) from single groundbased Doppler radar data remains a difficult task. The evolution and structure of landfalling TCs and their interactions with terrain are left uncharted due to the lack of dual-Doppler radar observations. Existing groundbased single-Doppler radar TC algorithms provide only qualitative information on axisymmetric TC center location and intensity. In order to improve understanding of the wind structures of landfalling TCs using the widely available WSR-88D data along the U.S. coastal region, a single ground-based radar TC wind retrieval technique, the ground-based Velocity Track Display (GBVTD) technique, is developed. Part I of this paper presents 1) single-Doppler velocity patterns of analytic, asymmetric TCs, 2) derivation of the GBVTD technique, and 3) evaluation of the GBVTD-retrieved winds using analytic TCs.

The Doppler velocity patterns of asymmetric TCs display more complex structure than their axisymmetric counterparts. The asymmetric structure of TCs can be inferred qualitatively from the pattern (or curvature) of the zero Doppler velocity line and the position and shape of the Doppler velocity dipole. However, without knowing the axisymmetric portion of the TC circulation, it is extremely difficult to extract quantitative information from these similar Doppler velocity patterns.

Systematic evaluations on the GBVTD-retrieved winds show good agreement compared with the original analytic wind fields for axisymmetric flows plus mean wind and/or angular wavenumber 1, 2, and 3 asymmetry. It is also shown that the GBVTD technique retrieves wind maxima that are not directly observed (perpendicular to the radar beams) because the GBVTD technique uses the Doppler velocity gradient, not the observed maxima, to retrieve wind maxima. The success of the GBVTD-retrieved winds and understanding their characteristics provide the theoretical basis to nowcast TC kinematic structure.
\end{abstract}

\section{Introduction}

Severe tropical cyclones ${ }^{1}$ (TCs) are one of the most devastating natural disasters along coastal regions in the tropics. Historically, severe TCs cause more loss of hu-

\footnotetext{
${ }^{1}$ An intense tropical cyclone where maximum winds exceed $34 \mathrm{~m}$ $\mathrm{s}^{-1}$ is known as hurricane, typhoon, or cyclone, depending on its geographical region.

* The National Center for Atmospheric Research is sponsored by the National Science Foundation.

+ Current affiliation: Wu-Fen-Shan Radar Station, Central Weather Bureau, Taipei, Taiwan.

\# Current affiliation: System Engineering Division, Institute for Information Industry, Taipei, Taiwan.
}

Corresponding author address: Dr. Wen-Chau Lee, National Center for Atmospheric Research, P.O. Box 3000, Boulder, CO 80307-3000. E-mail: wenchau@ucar.edu man life and property than all other natural disasters (Anthes 1982). Recent examples including Hurricane Iniki (1992), Hurricane Andrew (1992), Typhoon Herb (1996), and Typhoon Paka (1997) produced massive damage on the island of Kauai, southern Florida, Taiwan, and Guam, respectively.

A TC has long been recognized as a large cyclonic rotating body of winds characterized by a calm region near its circulation center. The intensity of a TC is usually classified by its surface maximum wind and/or minimum central pressure. Our understanding of TC circulations grew with the advance of atmospheric observing systems over the years, from rawinsondes, aircraft in situ measurements, dropsondes, satellite, and Doppler radars. Although the intensity of a TC can be inferred from one or more measurements from these instruments, the detailed three-dimensional kinematic structure of a TC can only be revealed by Doppler radars. 
Doppler radar has become an essential tool in observing mesoscale vortices since its first meteorological use in the late 1950s (Metcalf and Glover 1990). Since the early 1980s, the tail Doppler radar on the National Oceanic and Atmospheric Administration (NOAA) WP3D has collected Doppler radar data during hurricane reconnaissance and research missions. Dual-Doppler radar data can be collected from two perpendicular flight legs (e.g., a "figure 4" pattern) equipped with a classical (steerable) antenna or a single leg equipped with a dualbeam (i.e., the "French") antenna. Then, the three-dimensional TC internal structure can be reconstructed (e.g., Marks and Houze 1984, 1987; Marks et al. 1992; Roux and Viltard 1995). Although dual-Doppler radar derived TC structures have high research value, their real-time application capability is limited due to the elaborate data processing requirements.

A robust airborne single-Doppler wind retrieval technique, the velocity track display (VTD), was proposed to deduce the primary circulation of TCs in real time from an airborne Doppler radar scanning in a trackorthogonal mode (Lee et al. 1994). The VTD technique and its extension, extended VTD (EVTD; Roux and Marks 1996), run in real time on board the NOAA WP3Ds' workstations (Griffin et al. 1992). The VTD- and EVTD-derived winds are transmitted to the NOAA Tropical Prediction Center located in Miami, Florida, via satellite in near-real time, providing a critical piece of information to the forecasters.

In comparison, ground-based Doppler radars did not observe many TCs until recently and the dual-Doppler radar observations of TCs are still rare events because of the large baseline between Doppler radars. DualDoppler radar analyses are further restricted by the limiting domain that usually covers only a portion of a TC (e.g., Jou et al. 1997). Hence, single-Doppler radar techniques remain the primary tool for analyzing landfalling TCs. Single ground-based Doppler radar TC techniques have their roots in a more general mesocyclone Doppler radar signature. Through a pattern recognition approach, Donaldson (1970) found that a vortex produced a Doppler velocity dipole signature with opposite parity. This dipole Doppler velocity signature can be identified by a Doppler radar scanning azimuthally in a plane position indicator (PPI) mode. The azimuthal shear of radial velocities was used to identify potentially intense atmospheric vortices on the radar scope, which led to the tornado vortex signature (Brown et al. 1978).

The first application of the Doppler velocity vortex signature to tropical cyclones was proposed by Baynton (1979). The Doppler velocity pattern of a mean Atlantic hurricane (Shea and Gray 1973) was constructed by assuming a hypothetical coastal S-band (10-cm wavelength) Doppler radar with a $2^{\circ}$ beamwidth located 200 $\mathrm{km}$ away from the hurricane center. Baynton (1979) demonstrated that the Doppler velocity pattern of this hypothetical TC is essentially identical to those produced by mesocyclones.

Wood and Brown (1992, hereafter referred to as WB) systematically studied the Doppler velocity pattern of axisymmetric TCs consisting of both the tangential and radial flows based on a Rankine (1901) vortex. Quantitatively, a Rankine combined vortex (e.g., Lemon et al. 1978) is characterized by two flow regimes, a solidbody rotation, with $V_{T}$ proportional to $R$ (radius), surrounded by a potential vortex, with $V_{T}$ proportional to $1 / R$. The maximum wind, $V_{\max }$, is at the radius of maximum wind $\left(R_{\max }\right)$, the boundary between the regimes.

Wood and Brown (1992) concluded the following Doppler velocity characteristics for axisymmetric TCs.

1) Single-Doppler velocity patterns of an axisymmetric rotation and/or divergence vary as a function of an aspect ratio,

$$
\alpha=\frac{\text { core diameter }}{\text { distance from the radar to the TC center }} .
$$

2) The Doppler velocity maxima rotate counterclockwise (clockwise) as axisymmetric outflow (inflow) superimposed on an axisymmetric rotational flow. The magnitude of the axisymmetric rotation and radial flows can be estimated from the displacement of the extreme Doppler velocities.

3) The apparent TC center (defined as the midpoint of the chord connecting two extreme positive and negative Doppler velocity maxima) approaches the true TC center when $\alpha \rightarrow 0$.

4) The true TC center always falls on a circle passing through the radar and two Doppler velocity extremes.

The work by Baynton (1979) and WB provide the basis of using pattern recognition to estimate the intensity and center position of an axisymmetric TC, which are valuable resources for forecasters in making decisions in real time. Asymmetric TCs, however, have been commonly observed by reconnaissance aircraft since 1950s (e.g., LaSeur and Hawkins 1963; Hawkins and Rubsam 1968; Marks and Houze 1987; Marks et al. 1992). Stewart and Lyons (1996) first documented the asymmetric Doppler velocity patterns in Typhoon Ed (1993) from a Weather Surveillance Radar-1988 Doppler (WSR-88D) in Guam. Nevertheless, the general characteristics of Doppler velocity patterns in asymmetric TCs remain unclear. Therefore, it is beneficial to expand the analytic Doppler velocity patterns for axisymmetric TCs in WB to include asymmetric TCs and examine their properties. Although pattern recognition is an efficient and practical way in nowcasting TCs, it usually uses only point values, such as zero and peak Doppler velocities, to estimate TC center and intensity qualitatively. There is currently no ability to reconstruct the horizontal TC primary circulations from singleDoppler PPI scans using a pattern recognition method.

In developing the velocity-azimuth display (VAD), Lhermitte and Atlas (1962) first used all available Dopp- 
ler velocities in a PPI scan, in conjunction with a linear flow model, to yield quantitative information about weather events with winds varying linearly in space (also Caton 1963; Browning and Wexler 1968; Matejka and Srivastava 1991). Using a similar concept, but assuming a circular wind model, the VTD and EVTD techniques reconstructed the three-dimensional primary circulation of a TC from single airborne Doppler radar data. The axisymmetric and asymmetric tangential wind structures of Hurricane Gloria (1985) and Hugo (1989) were retrieved and compared favorably with in situ measurements.

Airborne Doppler radar observations are limited by the flight duration and operational restrictions over land. Therefore, airborne Doppler radar is more suitable to observe TCs over the open ocean. The completion of the WSR-88D network along the U.S. coastal region provides continuous surveillance capability of TC activity up to $\sim 400 \mathrm{~km}$ (low-level reflectivity factor only) and within $\sim 150 \mathrm{~km}$ (reflectivity and Doppler velocity) of the coastline. The WSR-88Ds offer a powerful tool for monitoring landfalling TCs should a VTD-type technique be implemented. Owing to different scanning geometry between the airborne and ground-based Doppler radar, a more general form of the equations is required to accommodate a more complex geometrical relationship. This new form of equations is named the groundbased VTD (GBVTD) technique.

Part I of this paper establishes the background and tools to retrieve TC circulation from single groundbased Doppler radar data. We present the Doppler velocity patterns of analytic axisymmetric and asymmetric TCs, derive the GBVTD technique, and examine the characteristics of the GBVTD-retrieved primary TC circulation using analytic wind fields. Section 2 describes the underlying concepts and the formulation of the GBVTD technique, while section 3 presents the construction of an analytic dataset. Section 4 discusses the Doppler velocity signatures of analytic asymmetric TCs. The general characteristics, advantages, and limitations of the GBVTD-retrieved analytic TC circulation are shown in section 5 . The aliasing of the asymmetric radial flow into tangential flow is discussed in section 6 and the conclusions in section 7 .

\section{The GBVTD technique}

\section{a. Geometrical relationship}

A ground-based Doppler radar $^{2}$ is fixed in space in contrast to an airborne Doppler radar, which moves in space. Therefore, the GBVTD geometrical relationship illustrated in Fig. 1 is different from the VTD geometrical relationship shown in Lee et al. (1994). Figure 1

\footnotetext{
${ }^{2}$ Hereafter, "Doppler radar" represents "ground-based Doppler radar" throughout this paper unless stated otherwise.
}

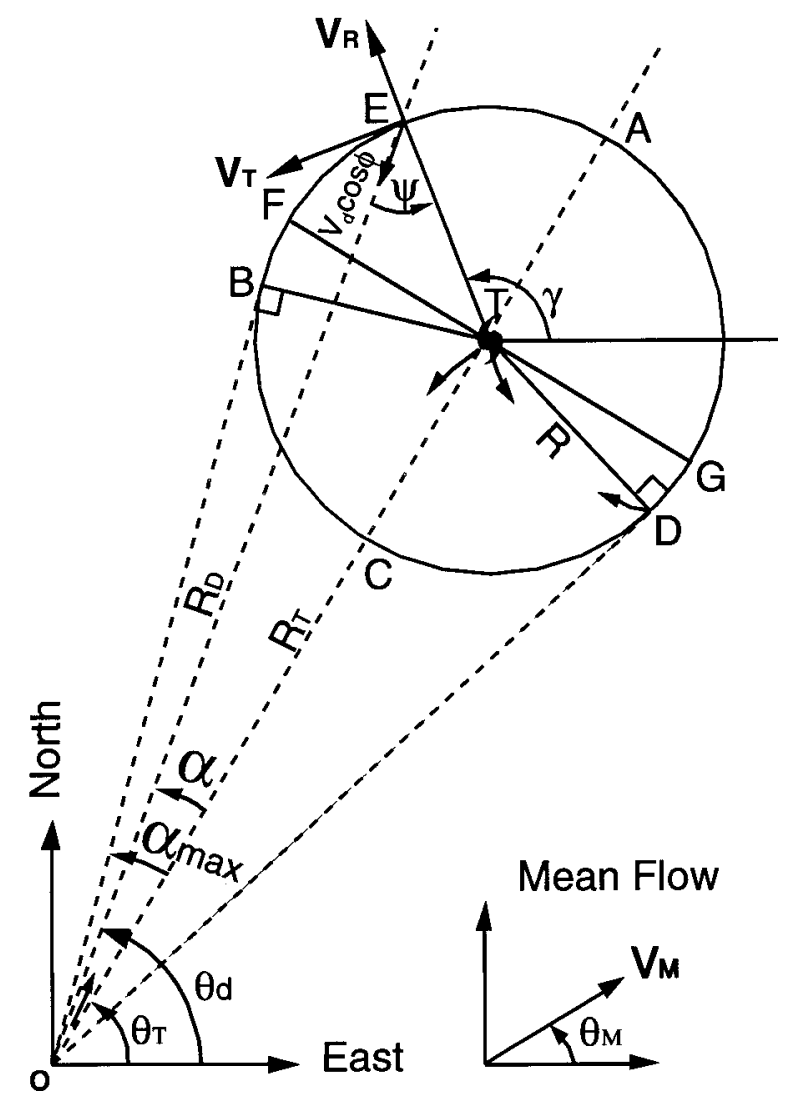

FIG. 1. The GBVTD geometry and symbols.

closely follows Fig. A1 in WB with modifications on several symbols and the use of a right-handed coordinate systems. Symbols and their definitions appearing in Fig. 1 are summarized in appendix A.

\section{b. Concept}

The GBVTD concept is illustrated in Fig. 2, which takes advantage of the Doppler velocity signatures of atmospheric vortices (Donaldson 1970; Brown et al. 1978). The vortex circulation is assumed circular where zero $\gamma$ and $\psi$ are defined at A (Fig. 1). For an axisymmetric positive tangential wind (counterclockwise in the Northern Hemisphere), $V_{T}$, illustrated in Fig. 2a, the Doppler velocities on a GBVTD ring are zero at $\mathrm{A}(\psi$ $=0)$ and $\mathrm{C}(\psi=\pi)$ where the beam direction is perpendicular to the TC tangential winds. The Doppler velocities equal the tangential wind at $\mathrm{B}(\psi=\pi / 2)$ and $\mathrm{D}(\psi=3 \pi / 2)$, where the Doppler radar measures the full component of the tangential winds. Note that the functional dependence of TC circulation is now with $\psi$, not $\gamma$. As a result, $\hat{V}_{d} / \cos \phi$ (defined in the next section) reduced to $-V_{T} \sin \psi$. For an axisymmetric negative radial wind (inflow), $V_{R}$, illustrated in Fig. 2b, the Doppler velocities on the GBVTD ring are equal to the radial wind at $\mathrm{A}$ and $\mathrm{C}$ while zero Doppler velocities are ob- 

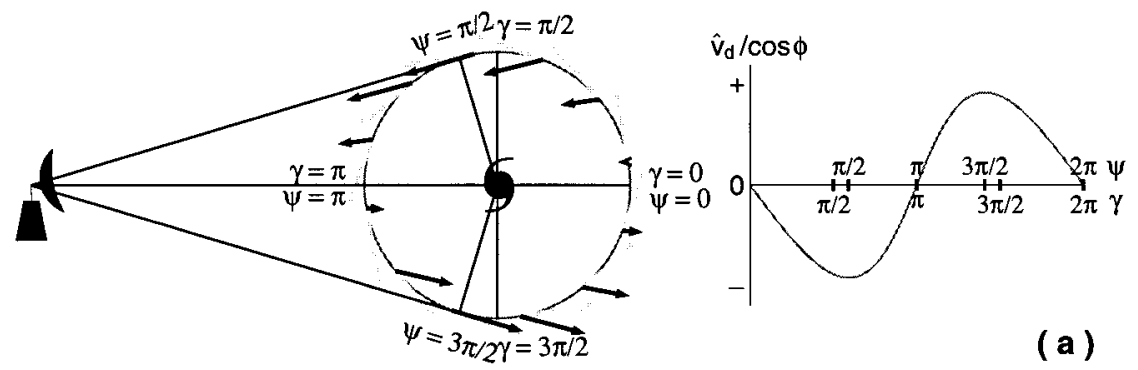

(a)
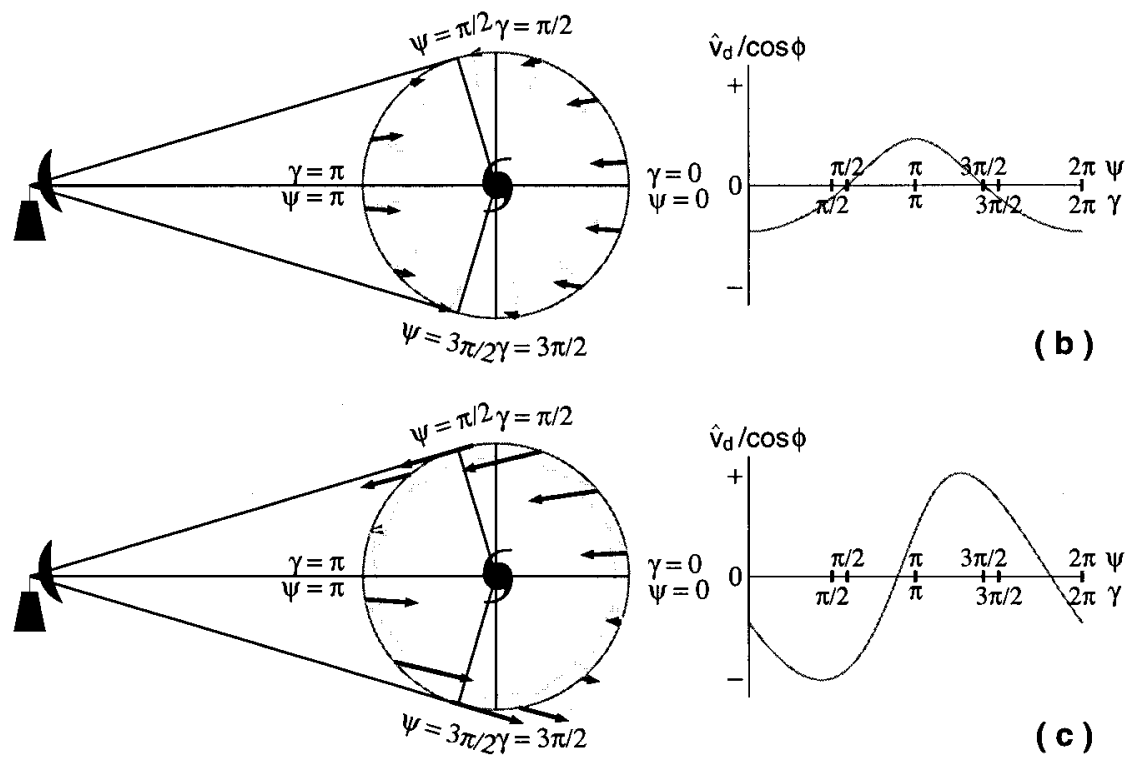

FIG. 2. GBVTD concept for (a) axisymmetric rotation, (b) axisymmetric radial inflow, and (c) axisymmetric rotation and radial inflow. Left panels illustrate the circulation of axisymmetric TCs (gray arrows) and their corresponding Doppler velocities (black arrows). Right panels illustrate the distributions of Doppler velocities vs azimuth angle.

served at B and D. So, $\hat{V}_{d} / \cos \phi$ has the form of $V_{R}$ $\cos \psi$. Combining these two axisymmetric flow patterns yields a negative sine curve with a phase shift (Fig. 2c). The sign of this phase shift indicates inflow (positive phase shift of maximum $V_{d}$ ) or outflow (negative phase shift of maxima $V_{d}$ ) while the magnitude of the phase shift is determined by $V_{R} / V_{T}$ of the vortex on a particular radius.

As discussed in Lee et al. (1994), the tangential and radial velocities in a real TC are usually asymmetric, composed of mean flow and waves of all scales. As a result, $\hat{V}_{d} / \cos \phi$ will not hold the simple sine and cosine form on a GBVTD ring as in the axisymmetric flow fields illustrated in Fig. 2 but will appear as a complex waveform that can be decomposed into Fourier components. These Fourier components contain contributions from the individual wavenumbers of the tangential and radial winds at each radius of a TC.

Note that the $\psi$ and $\gamma$ are identical only at $\mathrm{A}$ and $\mathrm{C}$, where $\sin \alpha_{\max }=0$. Here, $\sin \alpha$ is half of the aspect ratio $\alpha$ defined in WB. Due to the geometrical relationship in PPI scans as illustrated in WB (their Fig.
2), the Doppler velocity extreme are located at B and $\mathrm{D}(\psi=\pi / 2$ and $3 \pi / 2$, respectively), which do not coincide with $\mathrm{F}$ and $\mathrm{G}(\gamma=\pi / 2$ and $3 \pi / 2$, respectively) in Fig. 1. As a result, chord BD does not pass through the TC center (except when $\sin \alpha_{\max }=0$ ) that leads to distortions in the observed Doppler velocity patterns (Fig. 1 in WB).

The difference between the GBVTD and VTD techniques lies in the relationship between $\gamma$ and $\theta_{d}$ (F. Roux 1998, personal communication). In VTD, $\theta_{d}$ remains at $\pi / 2$ while $\gamma$ varies from 0 to $2 \pi$ on a constant radius because radar scans are considered parallel to each other. In GBVTD, $\theta_{d}$ and $\gamma$ are dependent because all beams in a PPI scan cannot be considered parallel. This difference implies that GBVTD contains more information than VTD but the inherent uncertainty is more complex (demonstrated in the next section). When $\sin \alpha_{\max } \approx 0$ (i.e., the vortex is far away from the radar or $R \ll R_{T}$ ), all beams in the PPI scan intersecting this ring can be treated as parallel beams and the GBVTD geometry reduces to the VTD geometry. Hence, B approaches F and D approaches G 
asymptotically. When $R \approx R_{T}$, B and D move toward $\mathrm{C}$ and coincide with $\mathrm{C}$ at the location of the radar $(\mathrm{O})$ when $\sin \alpha_{\text {max }}=1$. When $\sin \alpha_{\text {max }} \geq 1$, that is, the radar is either on or inside the GBVTD ring. As a result, the full component of $V_{T}$ is not observed by any beams but, instead, all Doppler velocities have significant contributions from the radial wind, $V_{R}$. When the ground-based radar is located at the vortex center $\left(\sin \alpha_{\max } \approx \infty\right)$, the geometrical relationship becomes essentially the VAD technique (Browning and Wexler 1968), which samples only the radial component of a vortex. Therefore, in order to sample the full tangential component of the vortex circulation at radius $R$ suitable for GBVTD analysis, a ground-based Doppler radar has to be located outside this radius (i.e., $R_{T}>R$ ).

\section{c. Mathematical formulation}

The Doppler velocity $\left(V_{d}\right)$, computed from the tangential velocity $\left(V_{T}\right)$, radial velocity $\left(V_{R}\right)$, vertical velocity $(w)$, terminal velocity $\left(v_{t}\right)$, and the mean flow $\left(V_{M}\right)$ of a tropical cyclone, is expressed by

$$
\begin{aligned}
V_{d}= & V_{M} \cos \left(\gamma-\theta_{M}\right) \cos \phi-V_{T} \sin \psi \cos \phi \\
& +V_{R} \cos \psi \cos \phi+\left(w-v_{t}\right) \sin \phi .
\end{aligned}
$$

The terminal velocity $\left(v_{t}\right)$ can be estimated from a $Z-v_{t}$ relationship. The vertical velocity $w$ is unknown and is set to zero in this study. After removing the contribution from $w$ and $v_{t}$ in (2), the remaining Doppler velocity is defined as $\hat{V}_{d}$ while its horizontal projection is expressed by

$$
\frac{\hat{V}_{d}}{\cos \phi}=V_{M} \cos \left(\gamma-\theta_{M}\right)-V_{T} \sin \psi+V_{R} \cos \psi .
$$

In order to perform a least squares fit, all terms on the right-hand side of (3) have to be expressed as a function of $\psi$ only. The term $\left(\gamma-\theta_{M}\right)$ in (3) can be written as $\theta_{T}+\alpha-\theta_{M}$. As a result,

$$
\begin{aligned}
& V_{M} \cos \left(\gamma-\theta_{M}\right) \\
& \quad=V_{M}\left[\cos \left(\theta_{T}-\theta_{M}\right) \cos \alpha-\sin \left(\theta_{T}-\theta_{M}\right) \sin \alpha\right] .
\end{aligned}
$$

of $\alpha_{\max }$ and $\psi$ (the derivation is provided in appendix $\mathrm{B}$ for interested readers) as follows:

$$
\begin{aligned}
& \cos \alpha \approx\left(\frac{1-\cos \alpha_{\max }}{2}\right) \cos 2 \psi+\left(\frac{1+\cos \alpha_{\max }}{2}\right) \\
& \sin \alpha=\sin \alpha_{\text {max }} \sin \psi .
\end{aligned}
$$

Substituting (4), (5), and (6) into (3), we obtain

$$
\begin{aligned}
& \frac{\hat{V}_{d}}{\cos \phi} \\
& \approx V_{M}\left[\cos \left(\theta_{T}-\theta_{M}\right)\left(\frac{1-\cos \alpha_{\max }}{2} \cos 2 \psi+\frac{1+\cos \alpha_{\max }}{2}\right)\right. \\
& \left.\quad-\sin \left(\theta_{T}-\theta_{M}\right) \sin \alpha_{\max } \sin \psi\right] \\
& \quad-V_{T} \sin \psi+V_{R} \cos \psi
\end{aligned}
$$

Since $\alpha_{\max }$ is a constant for a given radius, $\hat{V}_{d} / \cos \phi$ is a function of $\psi$ only. Following the VTD derivation in Lee et al. (1994), $\hat{V}_{d} / \cos \phi, V_{T}$, and $V_{R}$ can be written in terms of a truncated Fourier series:

$$
\begin{aligned}
\hat{V}_{d}(\psi) / \cos \phi & =\sum_{n=0}^{L}\left(A_{n} \cos n \psi+B_{n} \sin n \psi\right), \\
V_{T}(\psi) & =\sum_{n=0}^{M}\left(V_{T} C_{n} \cos n \psi+V_{T} S_{n} \sin n \psi\right), \\
V_{R}(\psi) & =\sum_{n=0}^{N}\left(V_{R} C_{n} \cos n \psi+V_{R} S_{n} \sin \psi\right),
\end{aligned}
$$

where $V_{T} C_{n}\left(V_{R} C_{n}\right)$ and $V_{T} S_{n}\left(V_{R} S_{n}\right)$ are the amplitude of the sine and cosine component of $V_{T}\left(V_{R}\right)$ for angular wavenumber $n$ (hereafter angular wavenumber is referred to as wavenumber).

Substituting (8), (9), and (10) to (7) and applying the following trigonometric identities,

$$
\begin{aligned}
2 \sin A \cos B & =\sin (A+B)+\sin (A-B), \\
2 \cos A \cos B & =\cos (A+B)+\cos (A-B), \\
2 \sin A \sin B & =\cos (A-B)-\cos (A+B),
\end{aligned}
$$

we obtain the following equation:

$$
\begin{aligned}
\frac{\hat{V}_{d}}{\cos \phi}= & \sum_{n=0}^{L}\left(A_{n} \cos n \psi+B_{n} \sin n \psi\right) \\
= & V_{M}\left[\cos \left(\theta_{T}-\theta_{M}\right)\left(\frac{1-\cos \alpha_{\max }}{2} \cos 2 \psi+\frac{1+\cos \alpha_{\max }}{2}\right)-\sin \left(\theta_{T}-\theta_{M}\right) \sin \alpha_{\max } \sin \psi\right]-V_{T} C_{0} \sin \psi \\
& +V_{R} C_{0} \cos \psi-\frac{1}{2} \sum_{n=1}^{M}\left\{V_{T} C_{n}[\sin (n+1) \psi+\sin (1-n) \psi]+V_{T} S_{n}[\cos (n-1) \psi-\cos (n+1) \psi]\right\} \\
& +\frac{1}{2} \sum_{n=1}^{N}\left\{V_{R} C_{n}[\cos (n+1) \psi+\cos (n-1) \psi]+V_{R} S_{n}[\sin (n+1) \psi+\sin (n-1) \psi]\right\} .
\end{aligned}
$$


The approximation sign has been replaced by an equal sign in (12) and in the rest of the equations in this paper. Matching corresponding coefficients of $\cos n \psi$ and $\sin n \psi$ in (12), we obtain

$$
\begin{aligned}
A_{0}= & \frac{1}{2}\left\{V_{M} \cos \left(\theta_{T}-\theta_{M}\right)\left(1+\cos \alpha_{\max }\right)\right. \\
& \left.\quad-V_{T} S_{1}+V_{R} C_{1}\right\} \\
A_{1}= & \frac{1}{2}\left(2 V_{R} C_{0}-V_{T} S_{2}+V_{R} C_{2}\right) \\
B_{1}= & \frac{1}{2}\left[V_{T} C_{2}-2 V_{T} C_{0}-2 V_{M} \sin \left(\theta_{T}-\theta_{M}\right)\right. \\
& \left.\quad \times \sin \alpha_{\max }+V_{R} S_{2}\right] \\
A_{2}= & \frac{1}{2}\left\{V_{M} \cos \left(\theta_{T}-\theta_{M}\right)\left(1-\cos \alpha_{\max }\right)\right. \\
& \left.\quad-V_{T} S_{3}+V_{T} S_{1}+V_{R} C_{3}+V_{R} C_{1}\right\} \\
A_{n}(n \geq 3)=\frac{1}{2}\left[V_{T} S_{n-1}-V_{T} S_{n+1}+V_{R} C_{n+1}\right. & \left.\quad+V_{R} C_{n-1}\right] \\
& \left.\quad+V_{R} S_{n-1}\right] .
\end{aligned}
$$

Equations (13)-(18) are the general expressions between the observed Doppler velocities and the tangential and radial winds on a GBVTD ring. For $n=0$ to $L$, there are $2 L+1$ equations but there are $4 L$ unknowns, $V_{M}, \theta_{M}, V_{T} C_{0}, V_{R} C_{0}, V_{T} C_{n}, V_{T} S_{n}, V_{R} C_{n}, V_{R} S_{n}$, for $n=$ 1 to $L-1$. Examination of the equations shows the cosine part of the tangential wind and the sine part of the radial wind of the same wavenumber have similar form and cannot be separated without additional observations. Therefore, this is an underdetermined problem and not all the coefficients can be uniquely determined from single-Doppler observations. In fact, there are many ways to close this underdetermined set of equations. One possible closure assumption is to follow the discussions in Lee et al. (1994), who assumed that the asymmetric $V_{R}$ is much smaller than the corresponding $V_{T}$. This is equivalent to assigning weighting function for all $V_{T}$ 's to 1 and all asymmetric $V_{R}$ 's to 0 , which reduces the numbers of unknowns from $4 L$ to $2 L+1$. Only those terms in (13)-(18) that do not include asymmetric $V_{R}$ are retained after the simple tropical cyclone model is applied.

When $L$ is limited from 0 to 4 and the above simple TC model is applied, the unknowns are reduced to 9 and can be solved in terms of $A_{n}$ and $B_{n}$ as follows:

$$
\begin{aligned}
V_{M} \cos \left(\theta_{T}-\theta_{M}\right)= & A_{0}+A_{2}+A_{4}-V_{R} C_{1}-V_{R} C_{3} \\
V_{T} C_{0}= & -B_{1}+B_{3}-V_{M} \sin \left(\theta_{T}-\theta_{M}\right) \\
& \times \sin \alpha_{\max }+V_{R} S_{2}
\end{aligned}
$$

$$
\begin{aligned}
V_{R} C_{0}= & A_{1}+A_{3}-V_{R} C_{2} \\
V_{T} S_{1}= & A_{2}-A_{0}+A_{4} \\
& +\left(A_{0}+A_{2}+A_{4}-V_{R} C_{1}-V_{R} C_{3}\right) \\
& \times \cos \alpha_{\max }+V_{R} C_{3} \\
V_{T} C_{1}= & -2\left(B_{2}+B_{4}\right)+V_{R} S_{1}+V_{R} S_{3} \\
V_{T} S_{2}= & 2 A_{3}-V_{R} C_{2} \\
V_{T} C_{2}= & -2 B_{3}+V_{R} S_{2} \\
V_{T} S_{3}= & 2 A_{4}-V_{R} C_{3} \\
V_{T} C_{3}= & -2 B_{4}+V_{R} S_{3} .
\end{aligned}
$$

The ignored terms (involving $V_{R}$ on the right-hand side) are presented in (19)-(27) to illustrate the biases due to the closure assumptions.

The $V_{M} \cos \left(\theta_{T}-\theta_{M}\right)$ (hereafter referred as "alongbeam $V_{M}$ ") and $V_{M} \sin \left(\theta_{T}-\theta_{M}\right)$ (hereafter referred as "cross-beam $V_{M}$ ") are the component of $V_{M}$ parallel and perpendicular to $\overline{O T}$ and there is not enough information to further separate $V_{M}$ and $\theta_{M}$. The cross-beam $V_{M}$ in (20) is unknown and aliased into the mean tangential wind, $V_{T} C_{\mathrm{O}}$. The error is the greatest when $\theta_{T}-\theta_{M}=$ $\pm \pi / 2$. Since $V_{M} \sin \theta_{M}(z)$ is normally much smaller than the mean tangential wind $V_{T} C_{0}$, the uncertainty in $V_{T} C_{0}$ should be small except in a fast moving, weak TC. Substituting (19)-(25) into (9) and (10), we obtain the vortex tangential winds (up to wavenumber 3 ) and the mean radial wind (wavenumber 0 ).

\section{d. Computational procedure}

The computational procedure of the GBVTD technique includes the following five steps.

1) Interpolating from PPI to constant-altitude PPI (CAPPI).

2) Identifying the $\mathrm{TC}$ center.

3) Interpolating data from CAPPI to TC cylindrical coordinates $(\psi)$.

4) Performing GBVTD analysis (on $\psi$ coordinates) on each radius and altitude to obtain GBVTD coefficients.

5) Tropical cyclone wind field construction in $\gamma$ coordinates from the GBVTD coefficients.

A bilinear interpolation scheme (Mohr et al. 1986) is used in steps 1 and 3. There are many ways to define TC centers, such as, reflectivity, minimum pressure, and circulations. In this study, the TC center is defined as the circulation center and its location is known. How to objectively identify a TC center in real situations is beyond the scope of this paper and will be discussed in Part II. When the GBVTD analysis is performed on CAPPI data at different altitudes, the three-dimensional structure of a TC can be constructed. 


\section{Construction of the analytic dataset}

There are several ground-based Doppler radar datasets of tropical cyclones available for evaluating the GBVTD technique. However, using real data brings in other factors, such as uncertainty in center location, biases by unknown vertical velocities and terminal velocities, and unevenly distributed targets, that are not directly related to the performance of the GBVTD technique. Most important, the true wind field is unknown in a real dataset. For testing, we use an analytic dataset instead of a real dataset because of the following reasons. 1) The performance of the GBVTD technique can be evaluated against wind fields with any combination of $V_{T}, V_{R}$, and $V_{M}$. 2) The effects of real data on GBVTD performance can either be isolated and/or simulated in the analytic dataset.

The analytic dataset is based on a single-level, idealized Rankine combined vortex at sea level. The axisymmetric $V_{T}$ is constructed as

$$
\begin{array}{ll}
V_{T}=V_{\max }\left(\frac{R}{R_{\max }}\right), & R \leq R_{\max }, \\
V_{T}=V_{\max }\left(\frac{R_{\max }}{R}\right), & R>R_{\max },
\end{array}
$$

where $V_{\max }$ and $R_{\max }$ are set to $50 \mathrm{~m} \mathrm{~s}^{-1}$ and $20 \mathrm{~km}$, respectively. In real TCs, the $V_{T}$ profiles usually drop off much slower outside $R_{\max }$ than the prescribed Rankine vortex. The axisymmetric $V_{R}$ is constructed such that outflow (inflow) is inside (outside) $R_{\max }$ while $V_{R}$ is zero at the TC center, $R_{\max }$, and infinity. This pattern is similar to that documented in Jorgensen (1984a,b). Hence,

$$
\begin{array}{ll}
V_{R}=C_{1}\left[\left(R_{\max }-R\right) R\right]^{1 / 2}, & R \leq R_{\max } \\
V_{R}=-C_{2}\left(R-R_{\max }\right)^{1 / 2} R_{\text {max }} / R, & R>R_{\max } .
\end{array}
$$

Here, $C_{1}$ and $C_{2}$ are scale factors and are assigned to $0.1 \mathrm{~s}^{-1}$ and $3 \mathrm{~m}^{0.5} \mathrm{~s}^{-1}$, respectively, putting $V_{R}$ within a reasonable range. The asymmetric TC circulations are constructed by superimposing higher wavenumbers onto the basic axisymmetric circulation. The magnitude of each wavenumber is set to $10 \mathrm{~m} \mathrm{~s}^{-1}$ where its phase varies from 0 to $2 \pi / n$, where $n$ is the wavenumber. Here, $V_{M}$ has a fixed amplitude of $10 \mathrm{~m} \mathrm{~s}^{-1}$ and $\theta_{M}$ varies from 0 to $2 \pi$. The resulting idealized TC contains the simple Rankine combined vortex and combinations of the asymmetric $V_{T}, V_{R}$, and $V_{M}$.

Ordinarily, radar resolution in the azimuthal direction is degraded with increasing range from the radar owing to the widening of the radar beam. In this paper, however, the radar beam is considered to be infinitesimally narrow such that radar resolution is perfect at all ranges. Additionally, the simulation model assumes that the radar measurements are free of noise. This hypothetical Doppler radar has a 120-km effective range and a high effective Doppler velocity range where velocity aliasing is not considered. The Doppler radar is located at the grid origin $(0,0)$, which is $60 \mathrm{~km}$ south of the TC center $(0,60)$. This simplification does not reduce the generality of the results because 1) the Doppler velocity pattern of an axisymmetric TC depends only on $\sin \alpha_{\max }$, where $\sin \alpha_{\max }$ between 0 and 1 is simulated at different $R$ for a fixed $R_{T}$, and 2 ) the Doppler velocity pattern of an asymmetric TC also depends on $\theta_{T}$ and $\theta_{M}$ where both forms of the angle dependence can be simulated by varying the phase angle of the asymmetric components and $\theta_{M}$.

\section{Characteristics of Doppler velocity patterns of analytic tropical cyclones}

The characteristics of the Doppler velocity patterns for a number of analytic TCs are discussed in this section. These idealized Doppler velocity patterns reveal many useful guidelines that can be used to interpret real TCs. The discussions are focused on five commonly observed flow regimes: 1) Rankine combined vortex, 2) axisymmetric $V_{T}$ plus $V_{M}, 3$ ) axisymmetric $V_{T}$ plus wavenumber 1, 4) axisymmetric $V_{T}$ plus wavenumber 2 , and 5) axisymmetric $V_{T}$ plus wavenumber 3 . The asymmetric radial flows are not included in the discussion since they have the same signatures as asymmetric tangential flows at a $90^{\circ}$ phase difference. Their effect is discussed qualitatively in section 6 .

\section{a. Rankine combined vortex}

The flow fields, their amplitude, and Doppler velocity signatures of the Rankine combined vortex are illustrated in Fig. 3. The Doppler velocity patterns of the axisymmetric $V_{T}$ (Fig. 3e) confirms those characteristics shown in WB, including the opening of the Doppler velocity patterns on the far side of the vortex. Note that the zero Doppler velocity line (thick solid line) is a straight line that passes through the TC center at $(0,60)$. The Doppler velocity pattern for axisymmetric $V_{T}$ in Fig. 3c is the "basic pattern" that will be used to compare with Doppler velocity patterns of asymmetric TCs in subsequent subsections.

The Doppler velocity pattern of a realistic axisymmetric $V_{R}$ (Fig. 3d) is more complicated than the simple radial outflow pattern illustrated in WB. The thick zero Doppler velocity circle centered at the TC center represents the transition from inflow to outflow at the $R_{\max }$. Another zero Doppler velocity circle passing through the radar and the TC center represents the zero projection of $V_{R}$ along each radar beam.

Combining axisymmetric $V_{T}$ and $V_{R}$ (Figs. $3 \mathrm{a}$ and $3 \mathrm{~b}$ ), the resulting Doppler velocity pattern is shown in Fig. 4. The velocity dipole rotates clockwise (counterclockwise) outside (inside) the $R_{\max }$ as shown in WB (their Fig. 3) and illustrated in the GBVTD concept. The zero Doppler velocity line passes through the TC center that bulges clockwise (in the inflow region outside $R_{\max }$ and 

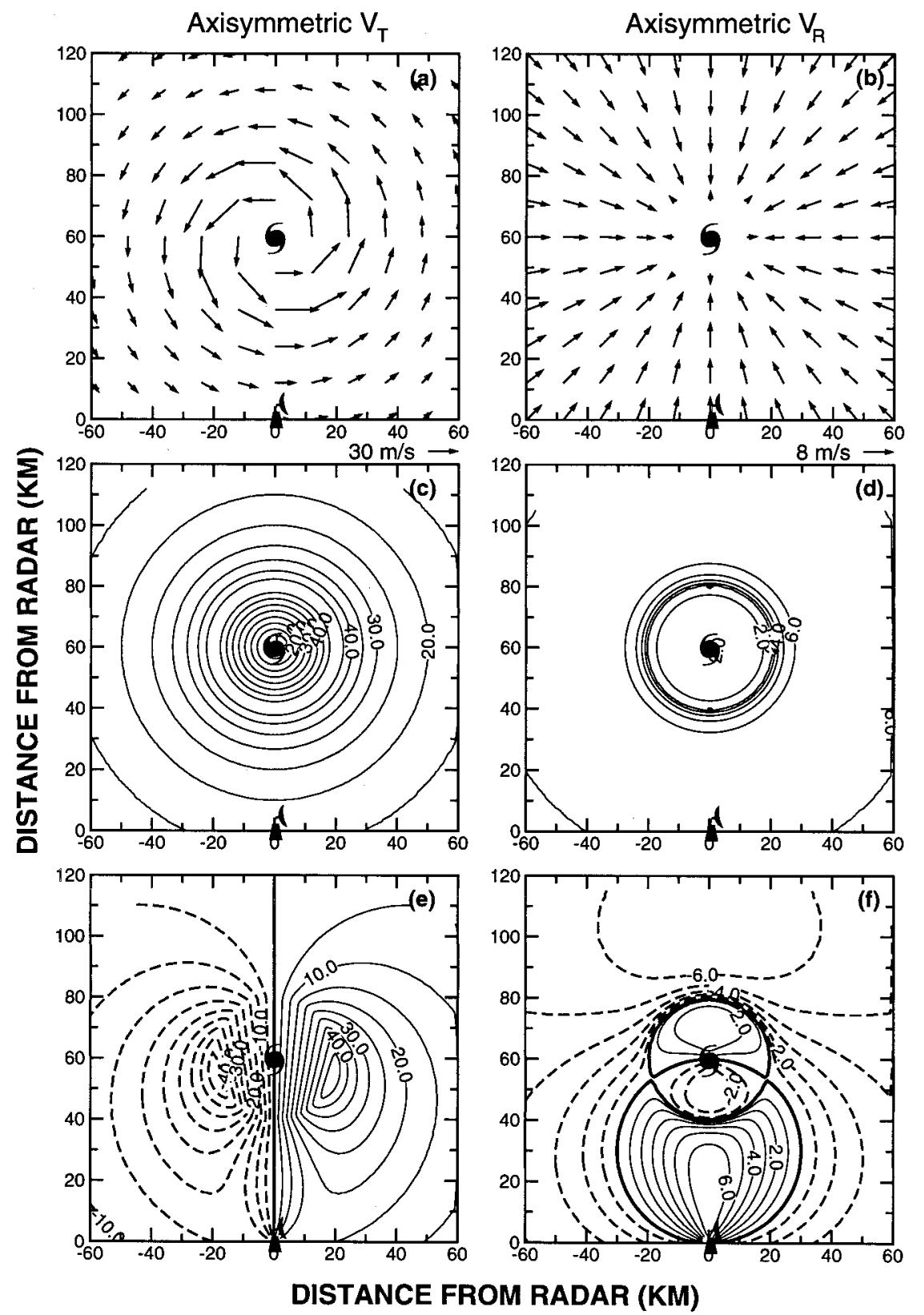

FIG. 3. (a) and (b) Axisymmetric $V_{T}$ and $V_{R}$ flow fields, (c) and (d) their amplitudes, and (e) and (f) the corresponding Doppler velocity patterns. Positive (negative) Doppler velocities are contoured in solid (dashed) lines. The thick solid line represents the zero Doppler velocity contour. The hurricane and radar symbols indicate the true TC center and the radar location, respectively.

counterclockwise (in the outflow region inside $R_{\max }$. The turning point from clockwise to counterclockwise bulging is located at the $R_{\max }$ where the inflow and outflow collide. This characteristic of the zero Doppler velocity line can be used in real time to identify the mean inflow/ outflow pattern and the location of maximum axisymmetric convergence/divergence on low-level PPI images.

\section{b. Effect of $V_{M}$}

When a $10 \mathrm{~m} \mathrm{~s}^{-1}$ northerly $V_{M}$ is superimposed on the basic pattern (Fig. 3c), the flow field changes (Fig. 5a) but the overall Doppler velocity pattern (Fig. 5b) remains the same (e.g., Brown and Wood 1991). The zero Doppler velocity line curves and shifts to the right side (cf. with Fig. 3b) of the true TC center $(0,60)$ in- 


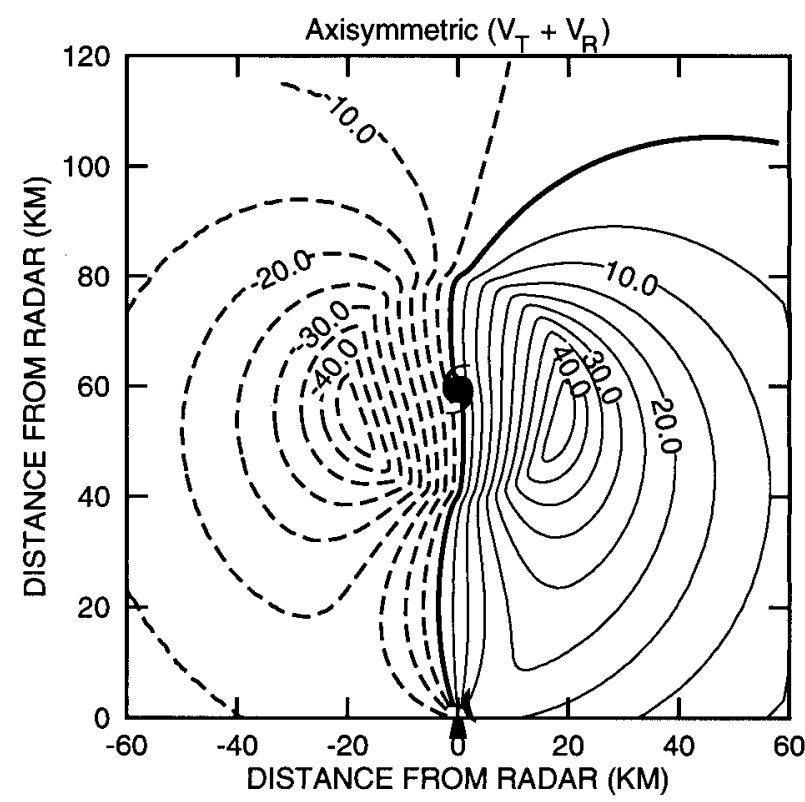

FIG. 4. The Doppler velocity pattern of axisymmetric $V_{T}$ plus $V_{R}$.

dicated by the hurricane symbol but passes through the apparent center. ${ }^{3}$ The straight Doppler velocity line goes through the true vortex center having the magnitude of the alongbeam $V_{M}$. The wind field and Doppler velocity pattern of a southerly $V_{M}$ (not shown) are essentially mirror images of Figs. 5a and 5b across the $x=0$ line.

The total winds with easterly (westerly) $V_{M}$ are shown in Fig. 5c (Fig. 5e) where the asymmetric flow fields shifts $90^{\circ}$ from their northerly (southerly) $V_{M}$ counterparts and the apparent center shifted southward (northward) of the true circulation center at $(0,60)$. Note that the zero line is a straight line through the true TC center when $V_{M}$ is perpendicular to the radar viewing direction.

Contrary to intuition, the Doppler velocity patterns in Figs. 5d and $5 \mathrm{f}$ are not mirror images of each other. Due to the fan beams in PPI scans, the cross-beam $V_{M}$ affects the Doppler velocities as a function of the beam angle. The primary difference resides in the peak magnitude of the Doppler velocity dipole. The westerly $V_{M}$ enhances (reduces) the basic flow south (north) of the TC center while easterly $V_{M}$ does the reverse. When $V_{M}$ is neither parallel nor perpendicular to the beam through the TC center (not shown), the Doppler velocity patterns possess a mixture of characteristics described above depending on the alongbeam and cross-beam $V_{M}$.

In summary, the effect of alongbeam $V_{M}$ essentially affects the characteristics of the zero Doppler velocity line (e.g., the location of the apparent center) while the crossbeam $V_{M}$ primarily influences the magnitude of the Dopp-

\footnotetext{
${ }^{3}$ The apparent center is defined as the point where ground-relative velocity is zero. This definition is different from the apparent center defined in WB.
}

ler velocity dipole. The zero Doppler velocity line curves clockwise (counterclockwise) on the far side of the TC center when the alongbeam $V_{M}$ is approaching (receding).

\section{c. Effect of wavenumber 1}

Figure 6 shows examples of the wavenumber 1 component located at azimuths $90^{\circ}, 180^{\circ}$, and $270^{\circ}$ superimposed on the basic flow. The zero Doppler velocity line remains a straight line and apparent centers collocated with the true vortex center at $(0,60)$ as the phase angle of wavenumber 1 peak wind rotates along $R_{\max }$. When wavenumber 1 is located at $90^{\circ}$ and $270^{\circ}$ (Figs. $6 \mathrm{~b}$ and $6 \mathrm{f}$ ), the Doppler velocity pattern is symmetric similar to the pattern of the basic pattern (Fig. 3b) while the difference is in the locations of the peak Doppler velocity dipole. A wavenumber 1 at $90^{\circ}\left(270^{\circ}\right)$ shifts the peak Doppler dipole north (south) of those in the basic pattern.

When wavenumber 1 is located at $180^{\circ}$ (Fig. 6d), the gradient toward the positive (negative) peak wind is tightened (loosened). The Doppler velocity pattern of wavenumber 1 located at $0^{\circ}$ is the mirror image of Fig. $6 \mathrm{~d}$ (not shown).

The total wind pattern produced by a northerly mean wind (Fig. 5a) and by a wavenumber 1 located at $180^{\circ}$ (Fig. 6c) are almost identical except for the locations of the apparent centers and the gradients of velocity in the cross-beam direction. Although these subtle differences are difficult to distinguish in the total wind fields, the differences in the corresponding Doppler velocity patterns are apparent in the behavior of the zero line and the cross-beam Doppler velocity gradients (Figs. 5b and $6 \mathrm{~d}$ ). It is important to compare those differences in Doppler velocity patterns in Figs. 5 and 6 that provide critical information for identifying not only the circulation center, but also the cause of the apparent wavenumber 1 structural asymmetry.

\section{d. Effect of wavenumber 2}

Figure 7 shows examples of the wavenumber 2 component superimposed on the basic pattern where two peak amplitudes are located at $90^{\circ}\left(270^{\circ}\right), 135^{\circ}\left(315^{\circ}\right)$, and $180^{\circ}\left(0^{\circ}\right)$. The total wind fields (left panels in Fig. 7) have an elliptical shape. The primary (minor) axis orients along the peaks (valleys) of the wavenumber 2 component. The apparent circulation centers are collocated with the true center at $(0,60)$ (e.g., Figs. 7 a, $7 \mathrm{c}$, and $7 \mathrm{e}$ ) while the zero Doppler velocity lines remain straight through the true TC center. When the wavenumber 2 is located at $90^{\circ}\left(270^{\circ}\right)$, the southern wind maximum pulls the the dipole toward the radar, while the northern wind maximum elongates the dipole on the far side of the TC center (e.g., Fig. 7b). When the wavenumber 2 is located at $0^{\circ}\left(180^{\circ}\right)$, the Doppler velocity pattern (Fig. 7f) is similar to the basic flow (Fig. 3b) except that the dipole is horizontally elongated (Fig. 


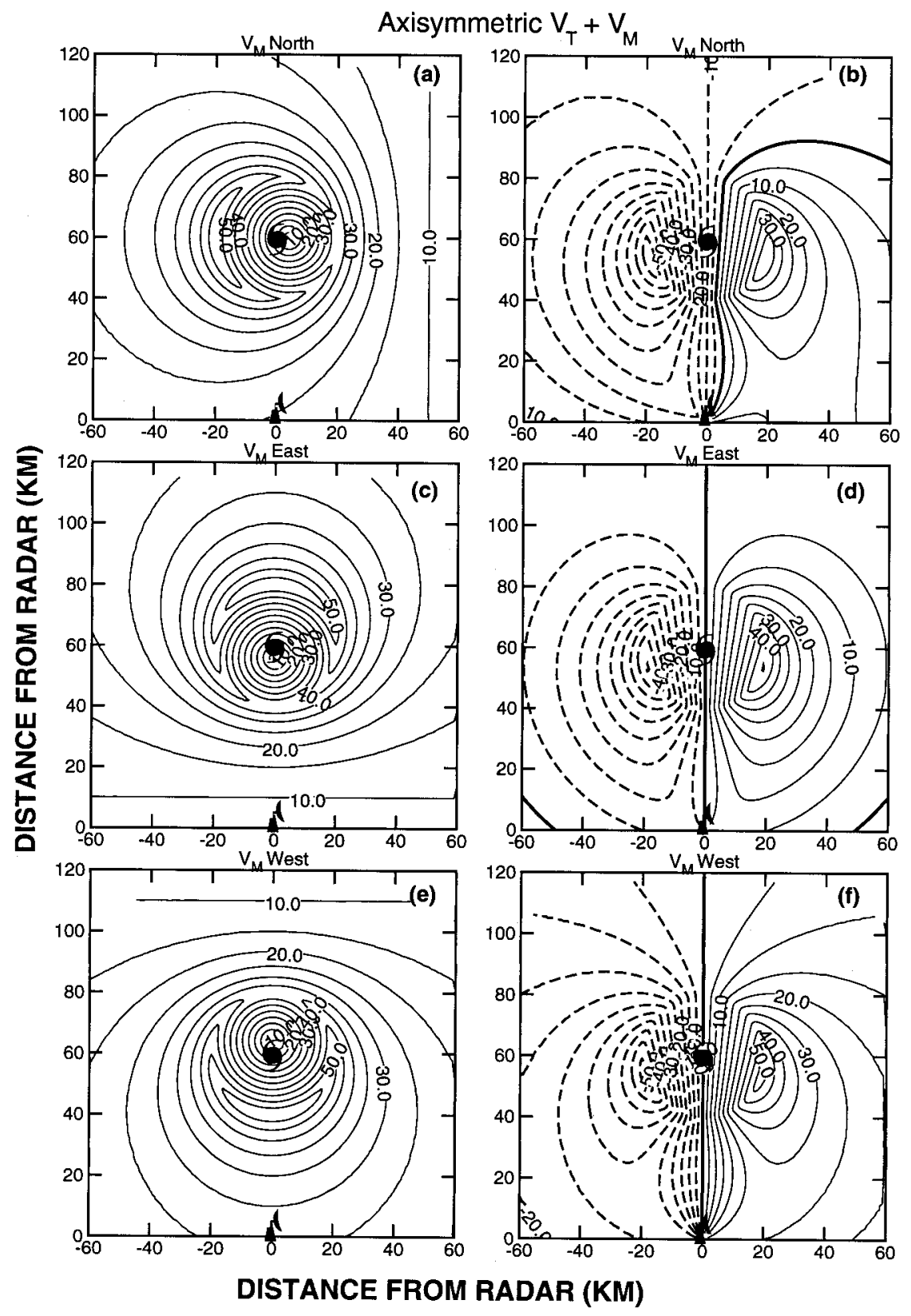

FIG. 5. The magnitude (left) and Doppler velocity pattern (right) of axisymmetric component of $V_{T}$ plus $V_{M}$ where (a) and (b) northerly $V_{M}$, (c) and (d) easterly $V_{M}$, and (e) and (f) westerly $V_{M}$.

7b). The chord connecting the dipole is not perpendicular to the zero Doppler velocity line for all wavenumber 2 phase angles (e.g., Fig. 7d) except for the two symmetric patterns illustrated previously. This characteristic distinguishes wavenumber 2 flows from axisymmetric and wavenumber 1 flows.

\section{e. Effect of wavenumber 3}

Examples of wavenumber 3 superimposed on the basic pattern at $90^{\circ}\left(210^{\circ}\right.$ and $\left.330^{\circ}\right), 150^{\circ}\left(270^{\circ}\right.$ and $\left.30^{\circ}\right)$, and $170^{\circ}\left(290^{\circ}\right.$ and $\left.50^{\circ}\right)$ are illustrated in Fig. 8. The apparent centers are collocated at the true vortex center $(0,60)$ where the straight zero Doppler velocity line passes through. The symmetric Doppler velocity patterns in Fig. $8 \mathrm{~b}$ are similar to Fig. $7 \mathrm{f}$ but these patterns are quite different between Figs. 8d and 7b. In general, the Doppler velocity patterns show an elongation on one side of the dipole (Fig. 8f) due to the asymmetry in wavenumber 3 flows. The cross-beam gradient of the Doppler velocities varies as the wavenumber 3 component advances in phase. 
(Axisymmetric + Wave 1) $\mathrm{V}_{\mathrm{T}}$

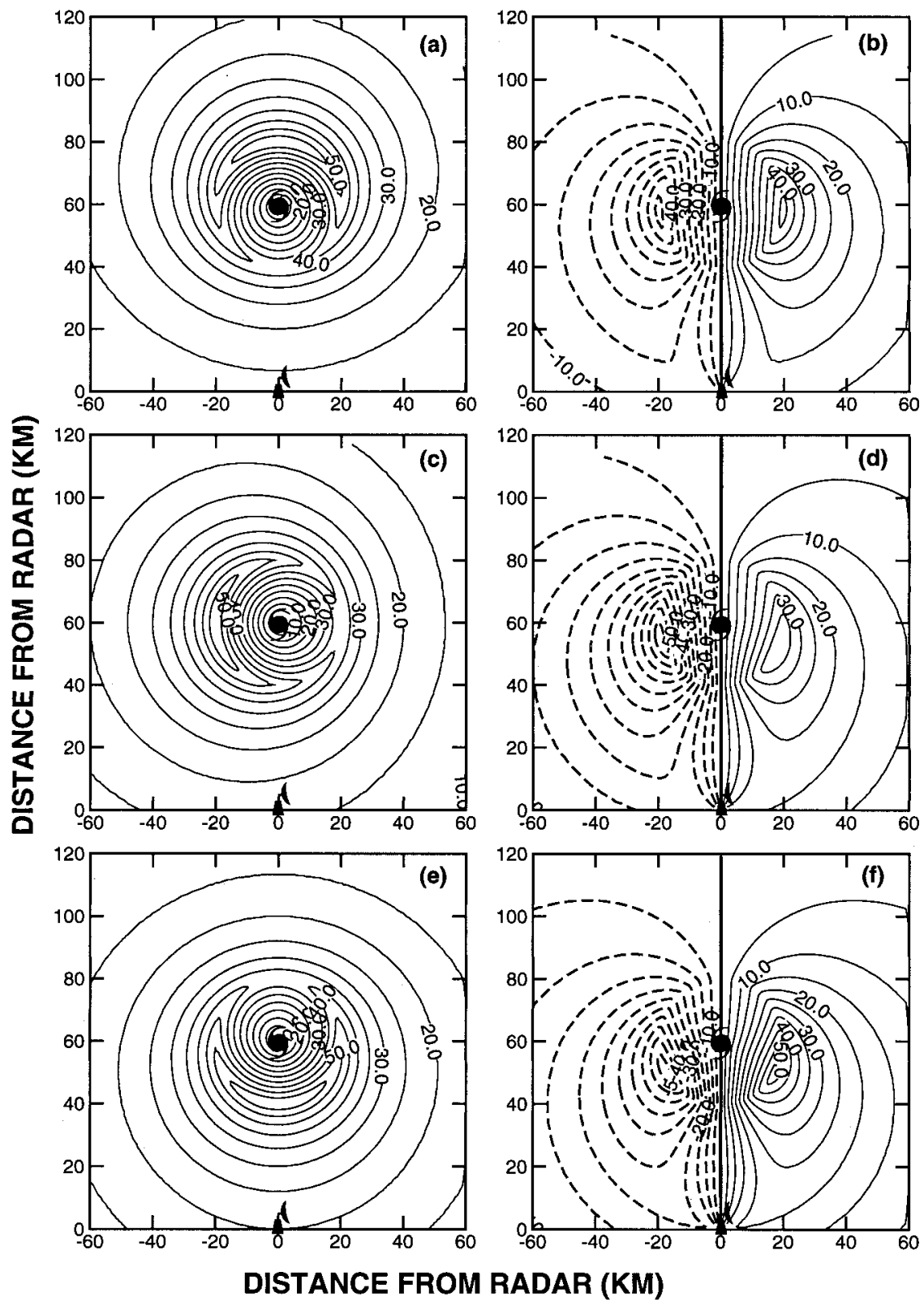

FIG. 6. The magnitude (left) and Doppler velocity pattern (right) of axisymmetric $V_{T}$ plus wavenumber 1 component of $V_{T}$ at (a) and (b) $90^{\circ}$, (c) and (d) $180^{\circ}$, and (e) and (f) $270^{\circ}$.

\section{The GBVTD-retrieved winds on analytic TCs}

The Doppler velocity patterns presented in the previous section provides qualitative guidance to identify TC structure from a low-level PPI scan. Although each asymmetric component possesses distinct characteristics in its Doppler velocity pattern, similar Doppler velocity patterns resulted from different combinations of asymmetry flow fields create difficulties in distinguishing TC structures exclusively from these Doppler velocity patterns. In this section, the GBVTD-retrieved circulations from these analytic TCs are presented to demonstrate that the GBVTD technique is robust in retrieving TC primary circulations from the Doppler velocity patterns. The GBVTD analysis is performed from $R=1 \mathrm{~km}$ to $R$ $=60 \mathrm{~km}$ with radial increment of $1 \mathrm{~km}$ and the data were interpolated onto 90 points on a constant radius ring with azimuthal increment of $4^{\circ}$. An error analysis is performed to document biases between the GBVTD-retrieved winds and their analytical counterparts. 
(Axisymmetric + Wave 2) $\mathrm{V}_{\mathrm{T}}$

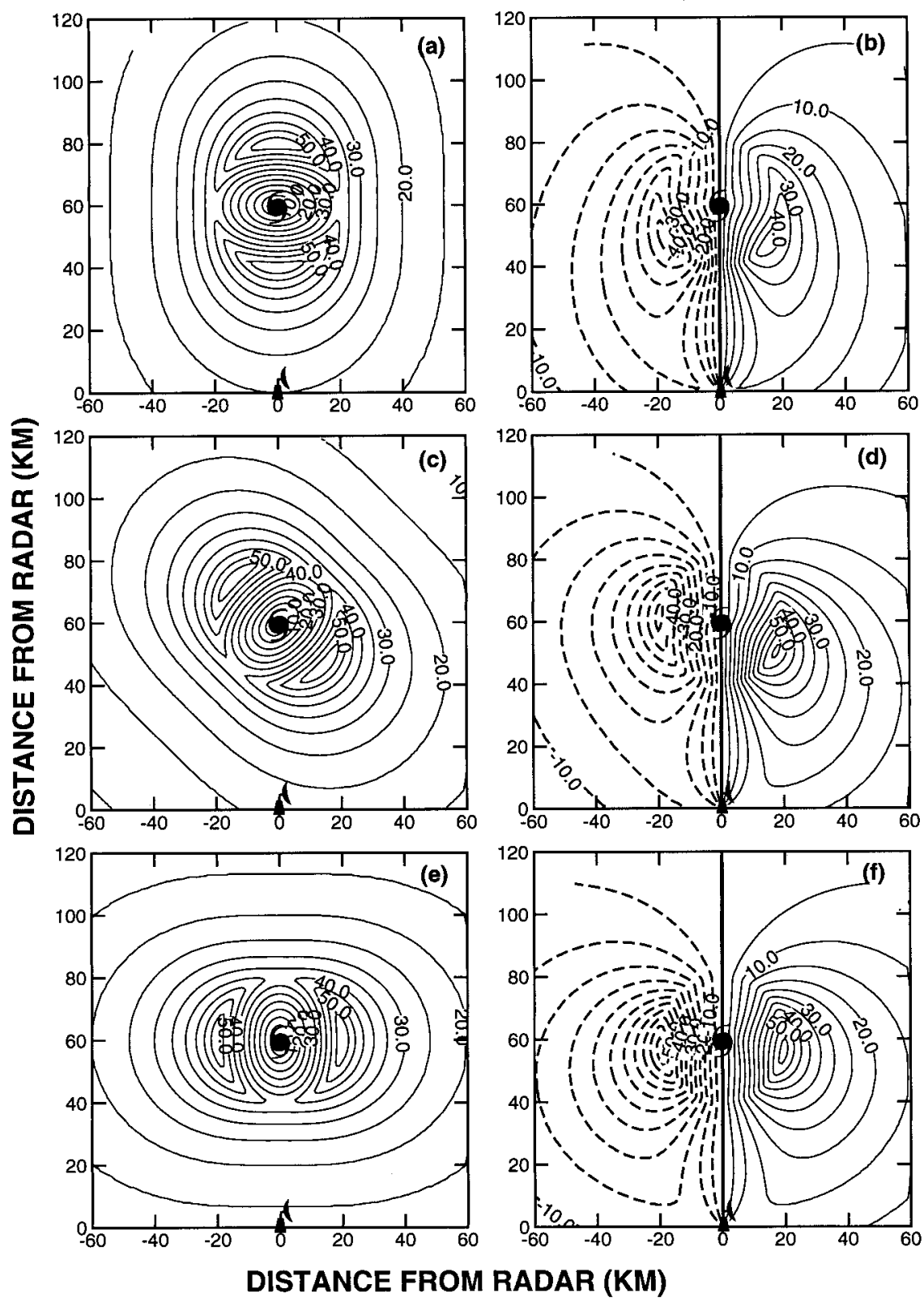

FIG. 7. The magnitude (left) and Doppler velocity pattern (right) of axisymmetric $V_{T}$ plus wavenumber 2 component of $V_{T}$ at (a) and (b) $90^{\circ}$, (c) and (d) $135^{\circ}$, and (e) and (f) $180^{\circ}$.

\section{a. Rankine combined vortex}

The GBVTD-retrieved $V_{T}$ (Fig. 9a) and $V_{R}$ (Fig. 9b) from the Doppler velocities in Fig. 4 are nearly identical compared with the analytic $V_{T}$ and $V_{R}$ in Figs. 3a and 3c. The bias (in \%) between the GBVTD-retrieved winds $\left(V_{T}\right.$ and $\left.V_{R}\right)$ and the analytic winds are less than $2 \%$ in all $\sin \alpha_{\max }$ and azimuth angles around the TC (Figs. 9c and 9d). These near-perfect results are not surprising because the axisymmetric circulation of $V_{T}$ and $V_{R}$ are explicitly resolved in the governing equations and illustrated in the GBVTD concept (Fig. 2).

\section{b. Basic pattern plus $V_{M}$}

When $V_{M}$ is northerly (alongbeam direction), the GBVTD-retrieved winds (Fig. 10a) reproduced their corresponding analytic winds (Fig. 5a). Some distortions exist at large $\sin \alpha_{\max }$; for example, the straight 10 $\mathrm{m} \mathrm{s}^{-1}$ contour (at $x=50 \mathrm{~km}$ ) in the analytic winds (Fig. 
(Axisymmetric + Wave 3) $\mathrm{V}_{\mathrm{T}}$

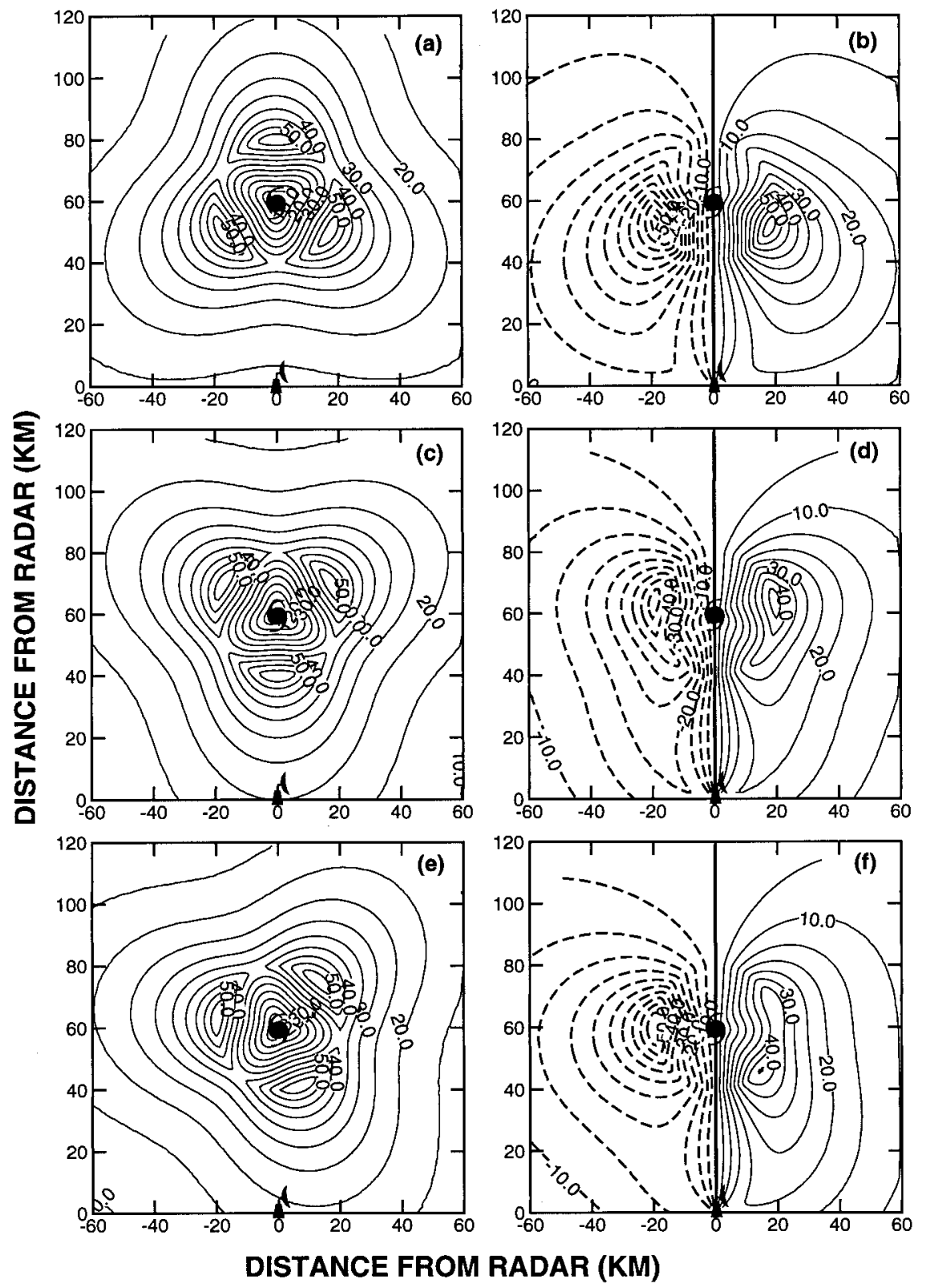

FIG. 8. The magnitude (left) and Doppler velocity pattern (right) of axisymmetric $V_{T}$ plus wavenumber 3 component of $V_{T}$ at (a) and (b) $90^{\circ}$, (c) and (d) $150^{\circ}$, and (e) and (f) $170^{\circ}$.

5a) becomes a curved contour in the retrieved wind field (Fig 10a). When $V_{M}$ has a cross-beam component (easterly or westerly in this example), the biases in the retrieved total winds become apparent and the GBVTD retrieves only the basic pattern while the asymmetry produced by $V_{M}$ is completely unrecoverable (comparing Figs. $10 \mathrm{~b}$ and $10 \mathrm{c}$ with Figs. 5c and 5e). The differences among the retrieved vortices in Figs. 10b,c and the basic vortex in Fig. 9c suggest that the cross-beam component of $V_{M}$ aliased into the retrieved $V_{T}$.
The systematic biases on the retrieved $V_{T}$ as a percentage of the $V_{M}$ on different $\sin \alpha_{\max }$ and $V_{M}$ angles are illustrated in Fig. 11a. Biases exist whenever a cross-beam $V_{M}$ component exists $\left(\theta_{M}\right.$ other than $0^{\circ}$ and $180^{\circ}$ ) and the error increases toward their maximum at $\theta_{M}=90^{\circ}$ and $270^{\circ}$. Also, as $\sin \alpha_{\max }$ increases, more of the cross-beam component is aliased into the retrieved axisymmetric $V_{T}$. The biases of the retrieved $V_{M}$ (Fig. 11b) are only a function of $\theta_{M}$. These characteristics can also be concluded from Eqs. (19) and 


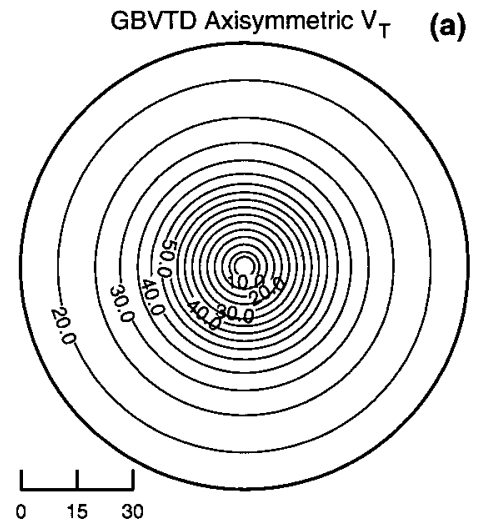

(a)
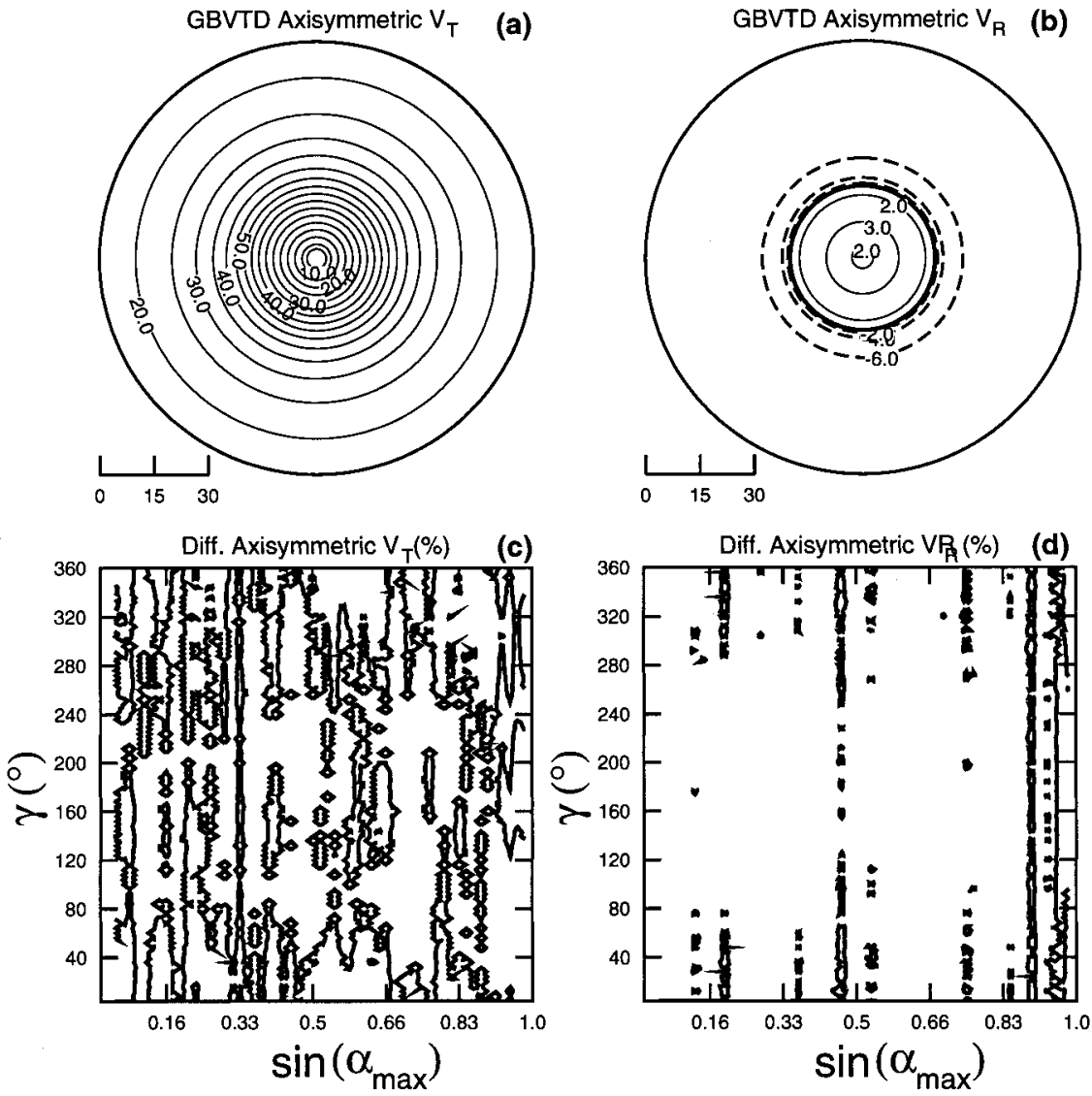

FIG. 9. The GBVTD-retrieved (a) axisymmetric component of $V_{T}$, (b) axisymmetric component of $V_{R}$, (c) error in retrieved component of $V_{T}(\%)$, and (d) error in retrieved component of $V_{R}(\%)$. The thick solid line is 0 contour. Positive (negative) contours are solid (dashed) lines. The contour interval for (c) and (d) is $1 \%$.

(20). These biases depend on the amplitude and direction of $V_{M}$.

\section{c. Basic pattern plus wavenumber 1}

The GBVTD-retrieved winds for the basic pattern plus wavenumber $1 V_{T}$ at different phases are illustrated in Fig. 12. Unlike the apparent wavenumber 1 asymmetry produced by $V_{M}$, the true wavenumber 1 asymmetry is retrieved by the GBVTD technique regardless of the phase angle of wavenumber 1 (comparing Figs. 12 and 6). Surprisingly, the GBVTD-retrieved wavenumber 1 structures at phase angle of $90^{\circ}$ (Fig. 12a) and $270^{\circ}$ (Fig. 12c) compared favorably with their analytic winds (Figs. 6a and 6e) where the Doppler velocity patterns show characteristics of axisymmetric vortices. These two cases are extremely challenging because the Doppler radar does not observe the peak amplitude directly (Figs. 6b and 6f). As a result, nowcasting TC properties based on the Doppler velocity pattern (Fig. $6 \mathrm{~b})$ will not only misplace the peak wind location but also underestimate its intensity. However, GBVTD re- trieves the wavenumber 1 structure via the observed Doppler velocity tendencies, not from the direct observation of the peak wind.

Although the characteristics of the wavenumber 1 structure is preserved in the GBVTD-retrieved wind field, it is evident that some distortions exist. These distortions are primarily due to performing least square fit on a nonlinear $(\psi)$ coordinate systems. In order to assess the systematic biases under these circumstances, the reconstructed wind fields were decomposed into the azimuthal $(\gamma)$ domain to compare with the analytic wind field. The biases in retrieved axisymmetric $V_{T}$ as a function of $\sin \alpha_{\max }$ and the phase $(\gamma)$ of wavenumber 1 are summarized in Fig. 13a. Only half of the domain from $90^{\circ}$ to $270^{\circ}$ phase angles are shown because of the symmetric nature of the biases.

Axisymmetric $V_{T}$ is overestimated (underestimated) when the peak wavenumber 1 is located to the north (south) of the TC center and the worst error (20\%) occurs when $\sin \alpha_{\max }=1$. The GBVTD technique always underestimates the wavenumber 1 amplitude. In contrast to the biases in axisymmetric $V_{T}$, the worst biases of 

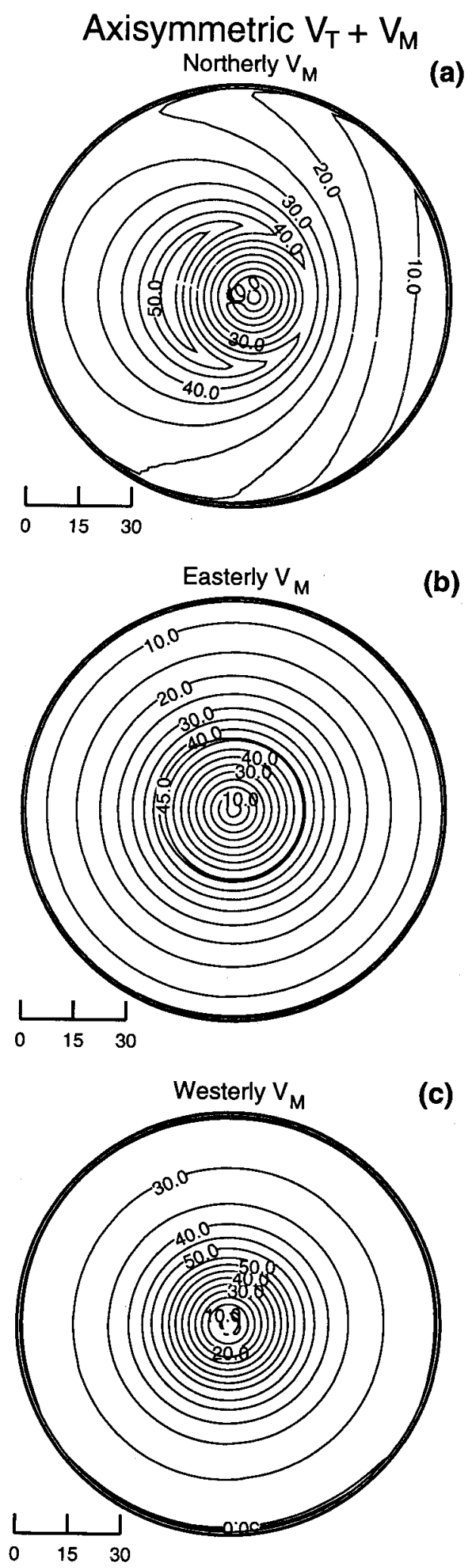

FIG. 10. The GBVTD-retrieved winds for axisymmetric component of $V_{T}$ plus $V_{M}$ where (a) northerly $V_{M}$, (b) easterly $V_{M}$, and (c) westerly $V_{M}$. the wavenumber 1 amplitude (Fig. 13b) occurs when the phase angles of the wavenumber 1 are $0^{\circ}$ and $180^{\circ}$, and the errors exceed $40 \%$ when $\sin \alpha_{\max }=1$ (e.g., the GBVTD ring passes through radar). The wavenumber 1 phase biases shift toward south (north) on the south (north) of the center with peaks at phase angles of $45^{\circ}$, $135^{\circ}, 225^{\circ}$, and $315^{\circ}$. Overall phase biases are small with maximum bias around $12^{\circ}$ when $\sin \alpha_{\max } \rightarrow 1$. In summary, the wavenumber 1 amplitude bias is worse than the biases in axisymmetric $V_{T}$ and phase of wavenumber 1 . If a $20 \%$ bias in the retrieved wind is acceptable, the GBVTD technique is able to reasonably retrieve wavenumber 1 asymmetric TCs when $\sin \alpha_{\max }$ $<0.7$.

\section{d. Basic pattern plus wavenumber 2}

The wavenumber 2 structures are well retrieved in all cases (cf. Figs. 14 and 7). The elliptical shape contours are preserved quite well when $\sin \alpha_{\max }<0.7$. However, the distortion outside $R_{\max }$ is apparent. The retrieved maximum wind closer to the radar is stronger and more elongated than the one away from the radar (e.g., Figs. 14a and 14b). Wavenumbers 1 and 3 of tangential winds have been generated in the retrieved winds due to aliasing where there was only a wavenumber 2 component in the analytical wind field. The GBVTD technique produces an artificial wavenumber 1 along the major axis of the ellipse (Fig. 14) where the peak (valley) is located closer to (away from) the radar. When comparing only the wavenumber 2 components, both the phase and amplitude of the retrieved component have errors compared with their analytic component. Nevertheless, retrieved wavenumbers 1 and 3 (from aliasing) compensate for most of the biases in retrieved wavenumber 2 to produce a plausible total wind field.

\section{e. Basic pattern plus wavenumber 3}

The GBVTD-retrieved winds for axisymmetric and wavenumber $3 V_{T}$ at different phase angles are illustrated in Fig. 15. The wavenumber 3 structure is recovered well inside $R_{\max }$ (e.g., $\sin \alpha_{\max }<0.33$ ). The peak amplitude of wavenumber 3 is severely reduced on the far side of the TC. However, the peak wind locations still compare well with their analytic counterparts (Figs. 8 and 15). Outside $R_{\max }$, only a wavenumber 2 pattern is apparent. As a result, only limited quantitative information of wavenumber 3 can be obtained from these severely aliased results beyond $\sin \alpha_{\max }>0.33$.

\section{Discussion}

When higher wavenumber tangential and radial flows are present in the idealized TC, systematic evaluation of errors in $V_{T}$ for each scenario becomes difficult. Examining (19)-(26) reveals that the higher wavenumber radial flow aliases into both amplitude and phase of their 

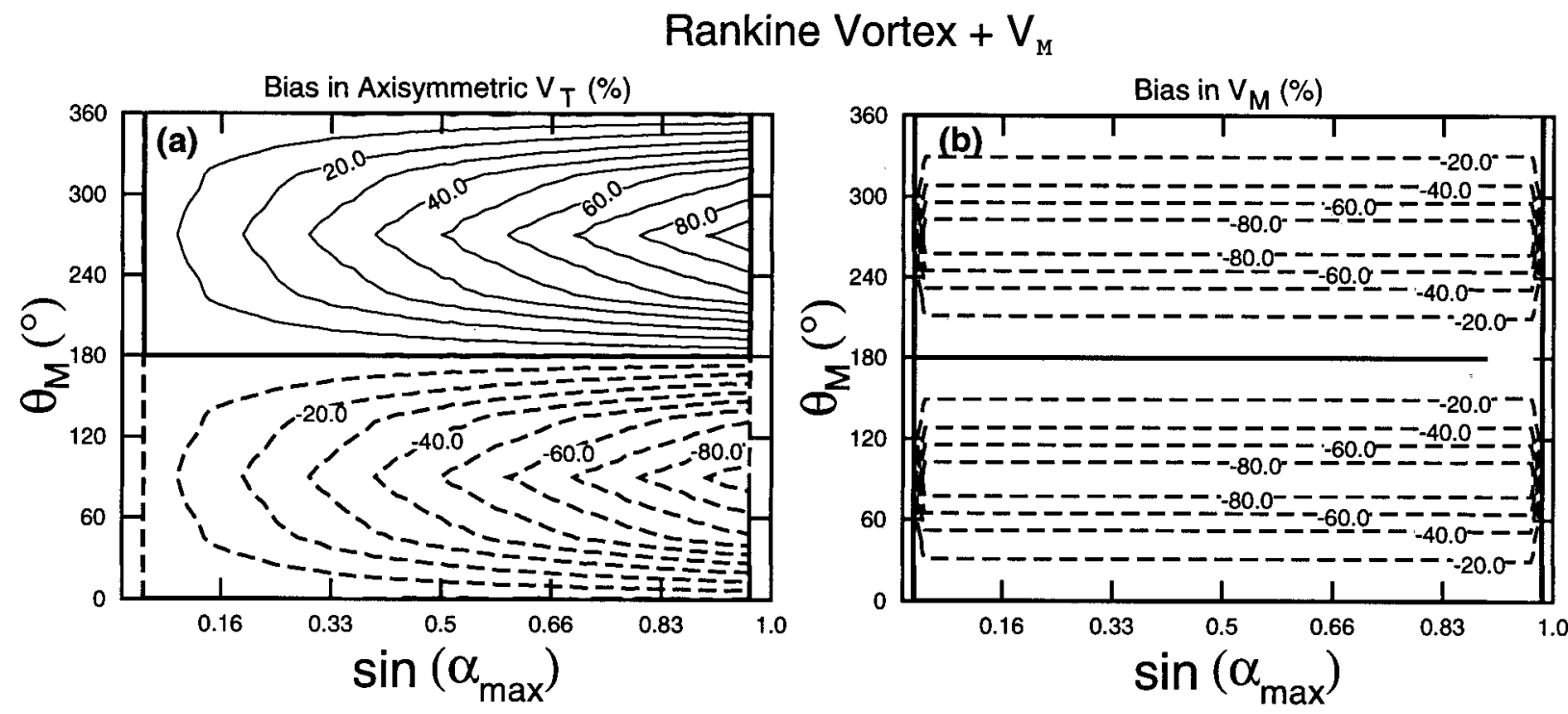

FIG. 11. Biases of the GBVTD-retrieved winds (\%) to the magnitude of $V_{M}$ as a function of $\sin \alpha_{\max }$ and $\theta_{M}$ : (a) axisymmetric $V_{T}$, and (b) $V_{M}$

tangential counterparts, as well as other wavenumbers owing to the nonlinear interaction among waves of different wavenumbers. For each wavenumber, the absolute error in the GBVTD-retrieved $V_{T}$ increases as the ratio of $V_{R} / V_{T}$ increases (not shown). There is no aliasing error when $V_{R} / V_{T}=0$. When both wavenumber $1 V_{T}$ and $V_{R}$ have equal amplitudes of $5 \mathrm{~m} \mathrm{~s}^{-1}$ (e.g., Roux and Marks 1996), the amplitude of the GBVTD-retrieved $V_{T}$ wavenumber 1 varies from 0 to $10 \mathrm{~m} \mathrm{~s}^{-1}$ while the phase varies between $\pm 90^{\circ}$, depending on the relative phases between wavenumber $1 V_{T}$ and $V_{R}$. Similar results can be extended to higher wavenumbers. For equal amplitudes of $V_{T}$ and $V_{R}$, the amplitude aliasing characteristics is the same as in the wavenumber 1 case, but the phase aliasing reduces from $\pm 90^{\circ}$ to $\pm 90 / \mathrm{n}^{\circ}$, because the pattern is repeated $n$ times around a circle for wavenumber $n$. When the amplitude of wavenumber $1 V_{R}$ is greater than $V_{T}$, the assumption of the GBVTD technique breaks down and the phase biases can be as large as $\pm 180^{\circ}$.

\section{Conclusions}

Part I of this paper presents two important aspects of using ground-based single-Doppler radar data in TC research and nowcasting. 1) Asymmetric TCs have more complicated Doppler velocity patterns than axisymmetric TCs and the qualitative storm structure can be identified from these patterns. 2) Introducing the GBVTD technique shows that the GBVTD technique is robust in estimating TC vortex structure from axisymmetric TC to wavenumber 3 asymmetry.

The Doppler velocity patterns summarized in this paper expand the scope to qualitatively identify TC structures from axisymmetric to asymmetric TCs using sin-
gle-Doppler radar data. These basic Doppler velocity patterns of asymmetric TCs form a useful database that enables forecasters to extract basic TC information from the Doppler velocity data in real time. The results show that asymmetric TC Doppler velocity patterns are significantly different from those of the axisymmetric TCs documented in WB. The zero Doppler velocity line passes through the true TC center in all cases except when the alongbeam mean flow $\left(V_{M}\right)$ exists and its shape is an important indicator of general structure of TCs. In addition, the zero Doppler velocity line becomes curved only when there are axisymmetric $V_{R}$ and alongbeam $V_{M}$. The differences in signatures are subtle but can be distinguished by examining the characteristics of the zero Doppler velocity contour.

The apparent wavenumber 1 asymmetry due to $V_{M}$ and the true wavenumber 1 asymmetry produce similar total wind patterns, but they can be distinguished by examining the characteristics of the zero Doppler velocity contour and the cross-beam Doppler velocity gradients across the TC center. The Doppler velocity patterns for wavenumbers 2 and 3 are quite distinct from the basic pattern and wavenumber 1 patterns and can be easily identified. The existence of asymmetric $V_{T}$ only modifies the cross-beam Doppler velocity gradient, but the zero Doppler velocity contour remains a straight line and passes through the true TC center.

The GBVTD technique provides an objective approach to estimate the primary circulation of TCs from single-Doppler velocity patterns. The GBVTD technique uses the least squares method to fit the primary TC circulation onto the Doppler velocities on a ring with constant radius from the TC center. The alongbeam $V_{M}$, axisymmetric $V_{R}$, and $V_{T}$ can be retrieved for each radius and altitude. The asymmetric radial flows 
(Axisymmetric + Wave 1) $V_{T}$

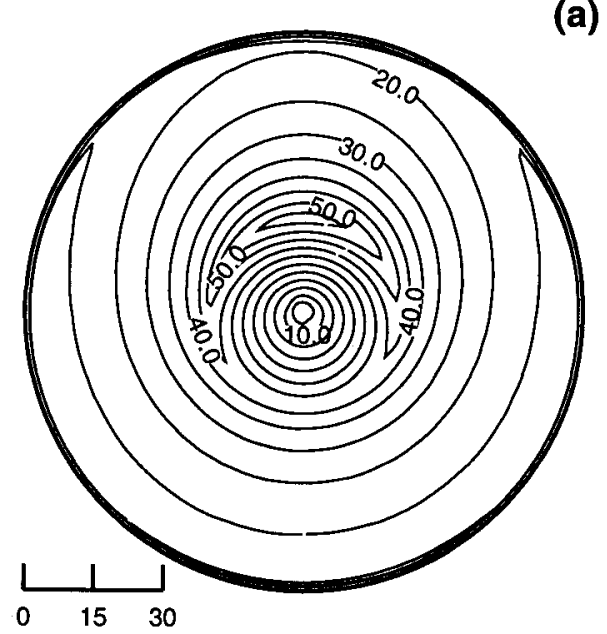

(b)

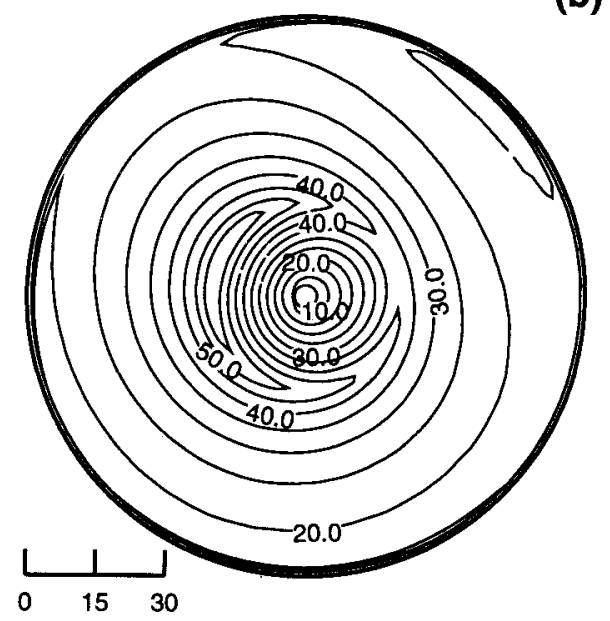

(c)

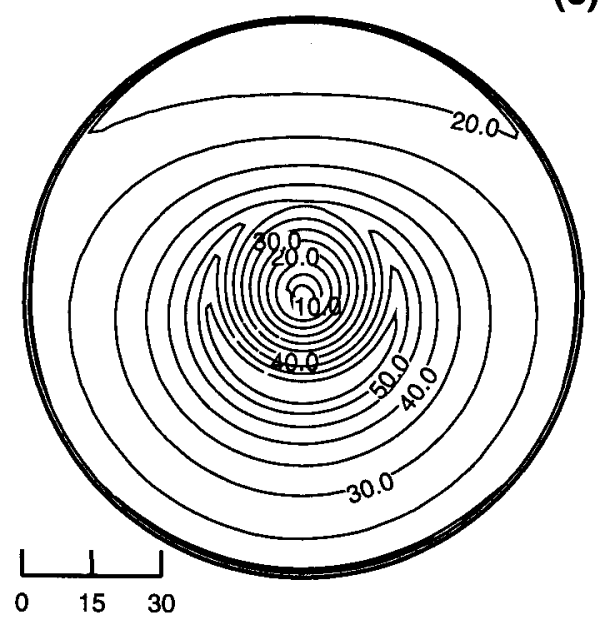

FIG. 12. The GBVTD-retrieved winds for axisymmetric $V_{T}$ plus wavenumber 1 component of $V_{T}$ at (a) $90^{\circ}$, (b) $180^{\circ}$, and (c) $270^{\circ}$.
Rankine Vortex + Wave 1
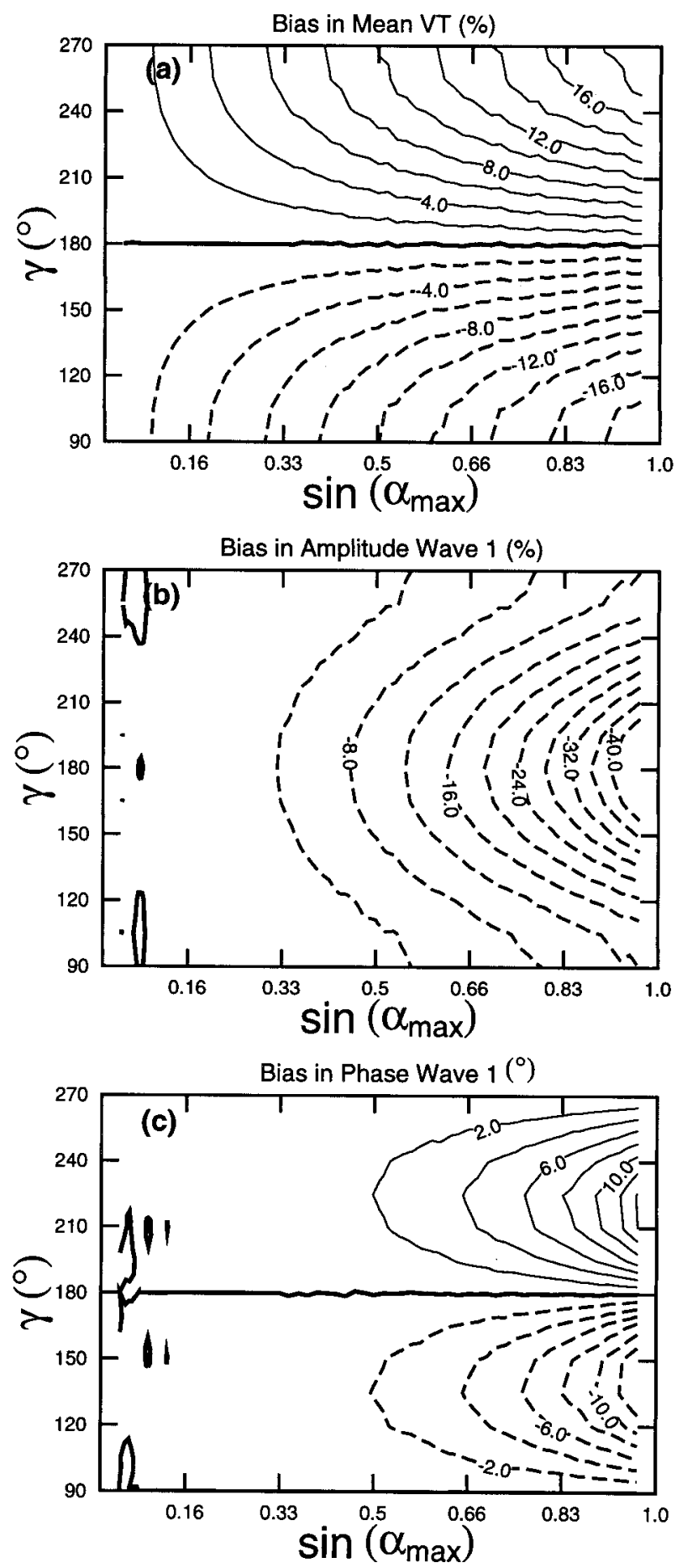

FIG. 13. Biases of the GBVTD-retrieved winds (\%) to their analytic values as a function of $\sin \alpha_{\max }$ and $\theta_{M}$ : (a) axisymmetric $V_{T}$, and (b) magnitude of wavenumber 1 , and (c) phase of wavenumber 1 . 
(Axisymmetric + Wave 2) $V_{T}$

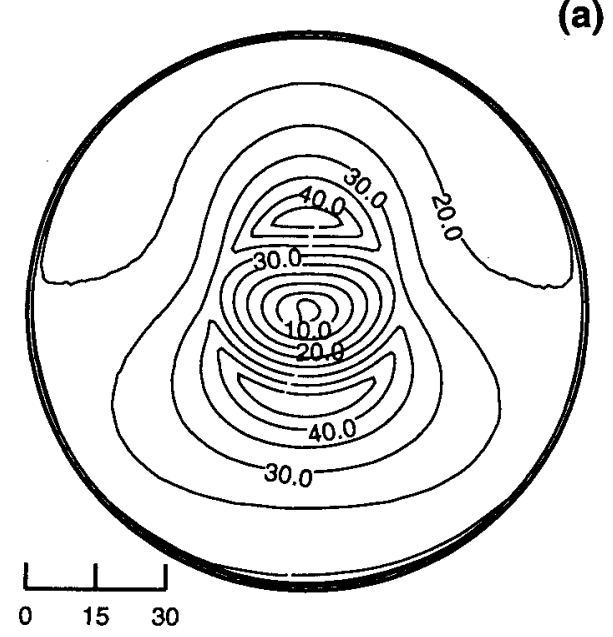

(b)

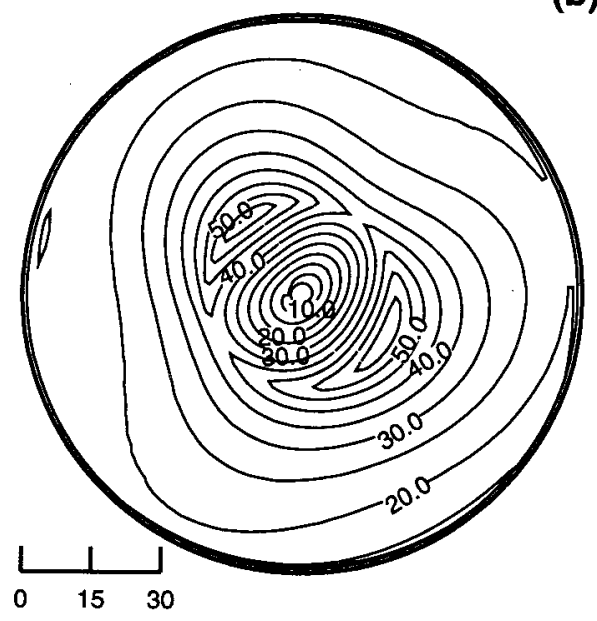

(c)

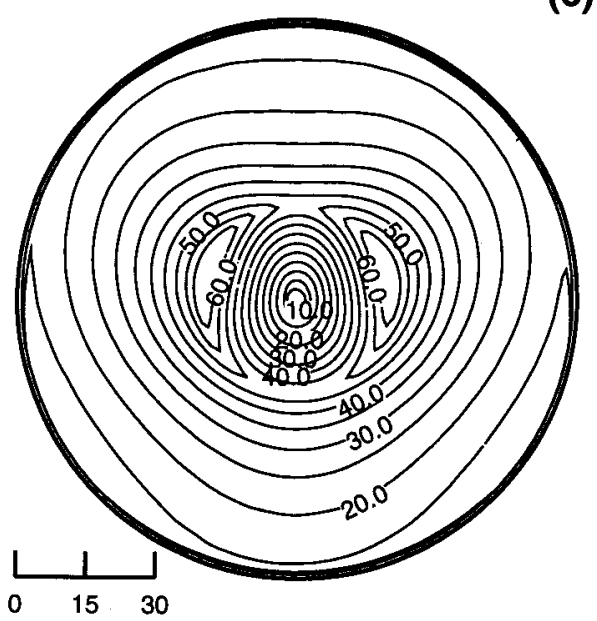

FIG. 14. The GBVTD-retrieved winds for axisymmetric $V_{T}$ plus wavenumber 2 component of $V_{T}$ at (a) $90^{\circ}$, (b) $135^{\circ}$, and (c) $180^{\circ}$.
(Axisymmetric + Wave 3) $V_{T}$

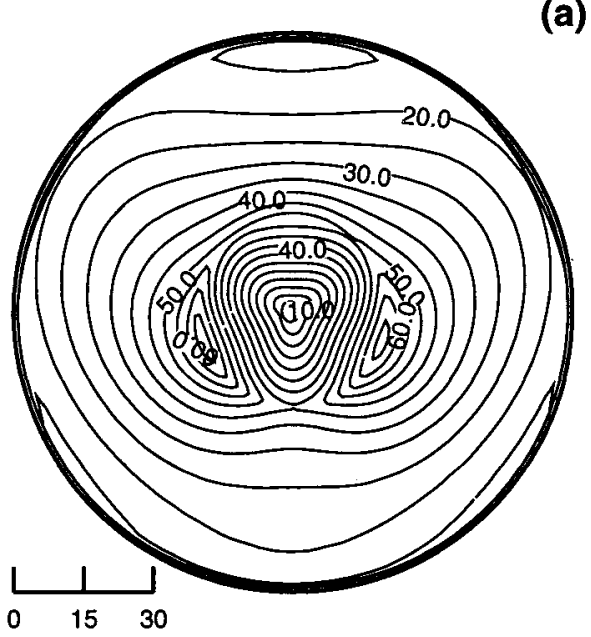

(b)

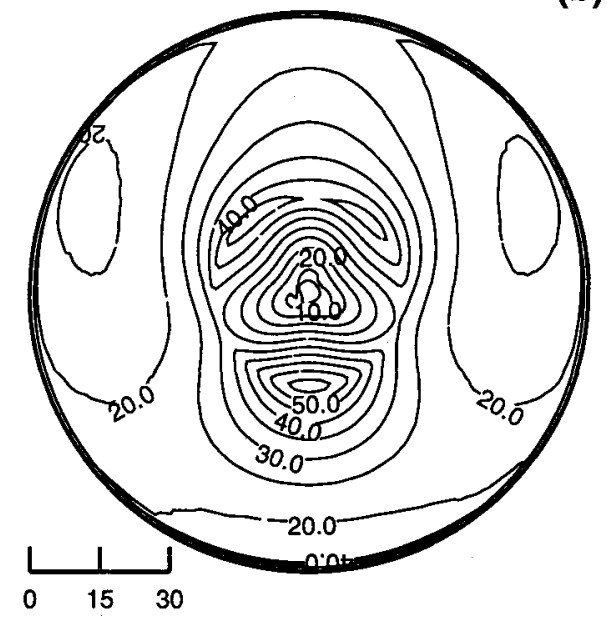

(c)

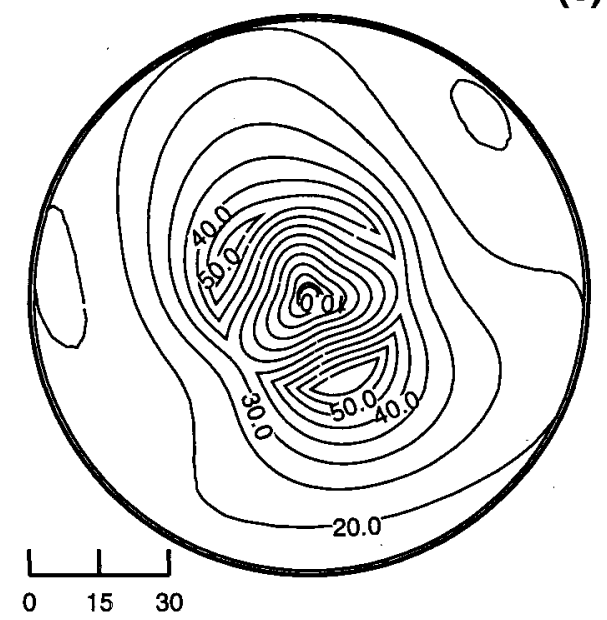

FIG. 15. The GBVTD-retrieved winds for axisymmetric $V_{T}$ plus wavenumber 3 component of $V_{T}$ at (a) $90^{\circ}$, (b) $150^{\circ}$, and (c) $170^{\circ}$. 
and the cross-beam $V_{M}$ are not resolved, but alias into the tangential winds and along-beam $V_{M}$. These quantitative TC structures cannot be retrieved from a pattern recognition method.

The GBVTD technique retrieves good total wind in nearly all cases tested in this study. It has been shown that the GBVTD technique is able to retrieve unsampled wind maxima that were not directly observed by a Doppler radar because it retrieves wind maximum using the tendency embedded in the Doppler velocity pattern, not relying on the observed absolute maxima or minima. This characteristic, in conjunction with the ability to identify basic TC structures, are the two most important advantages of using the GBVTD technique over the conventional pattern recognition methods in nowcasting landfalling TCs.

The quality of the GBVTD-retrieved wind fields strongly depends on $\sin \alpha_{\max }$ (the aspect ratio, $R / R_{T}$ ), which essentially determines the distortion of the GBVTD geometry owing to the proximity to the radar. The upper bond of $\sin \alpha_{\max }$ for good wind retrieval is further limited by the existence of asymmetric circulations. For a $20 \%$ bias, the upper limits of $\sin \alpha_{\max }$ are $0.7,0.5$, and 0.33 for wavenumbers 1,2 and 3 , respectively. These results form theoretical bases on how to interpret and apply the GBVTD results in future TC studies.

In Part II of this series (Lee and Marks, manuscript submitted to Mon. Wea. Rev.), an objective vortex center finding algorithm, the GBVTD-simplex algorithm, is presented. Applications of the GBVTD to real landfalling TCs are the focus in Part III of this series (Lee et al. 1999, manuscript submitted to Mon. Wea. Rev.), where the three-dimensional structure of Typhoon Alex (1987) is retrieved from the GBVTD technique using the circulation center deduced from the GBVTD-simplex algorithm.

Acknowledgments. The authors are grateful to Drs. Peter Hildebrand, Frank Marks, Frank Roux, Tammy Weckwerth; Mr. Peter Dodge; and two anonymous reviewers for their valuable comments that greatly improved this paper. Ms. Susan Stringer helped in preparing figures and Ms. Jenifer Delaurant proofread the manuscript. This research is partially supported by the National Science Foundation of the United States and the National Science Council of Taiwan, Republic of China, under Grant NSC 88-2111-M-002007-AP6.

\section{APPENDIX A}

\section{Coordinate Systems of the GBVTD Technique}

The coordinate systems and symbols of the GBVTD technique are illustrated in Fig. 1 elsewhere in the text. There are two coordinate systems: a cylindrical coordinate system centered at the TC and a radar spherical coordinate system centered at the radar. These coordinate systems and corresponding wind components are defined as follows.

O: the location of the ground-based Doppler radar T: the center of the TC

$\mathrm{R}$ : the radial distance from the TC center to the ring at a constant altitude where the GBVTD analysis is performed

E: the intersection of a radar beam and a constant radius ring

A, C: intersections of the radar beam (OT) and a ring of radius $\mathrm{R}$

$\mathrm{B}, \mathrm{D}$ : intersections of radar beams tangent to the ring of radius $\mathrm{R}$ where $\mathrm{OB} \perp \mathrm{TB}$ and $\mathrm{OD} \perp \mathrm{TD}$

F, G: $\quad$ FG passes through $\mathrm{T}$ and is perpendicular to OT

$R_{D}$ : the radial distance between the radar and a pulse volume

$R_{T}$ : the radial distance between the radar and the storm center

$\theta_{d}: \quad$ the mathematical angle of the radar beam measured counterclockwise from the east

$z$ : vertical axis of the TC cylindrical coordinate, $z=0$ is sea level

$\phi: \quad$ the elevation angle of the radar beam

$V_{T}$ : the tangential velocity of the TC, positive counterclockwise (clockwise) in the Northern (Southern) Hemisphere

$V_{R}$ : the radial velocity of the TC, positive outward from the TC center

$V_{d}$ : Doppler velocity from the ground-based Doppler radar, receding (approaching) velocities from the radar are positive (negative)

$v_{t}$ : terminal fall speed of the precipitation particles, positive is downward

$w$ : vertical velocity, positive is upward

$V_{M}(z)$ : the magnitude of the mean wind flow, which is a function of altitude

$\theta_{M}(z)$ : the direction of the mean wind flow, which is a function of altitude

$\alpha: \quad$ the angle subtended by OE and OT $(\angle \mathrm{TOE})$

$\alpha_{\max }: \quad$ the maximum $\alpha$ at a given radius $(\angle \mathrm{TOB})$

$\psi: \quad \quad \angle \mathrm{OET}$; when $\psi=0$ (A) and $\pi(\mathrm{C})$, the radar beam is parallel to radius TE; when $\psi=\pi / 2$ (B) and $3 \pi / 2$ (D), the radar beam is normal to radius $\mathrm{TE}$

$\gamma: \quad$ the mathematical angle for $\mathrm{E}$ in the TC cylindrical coordinate

$\theta_{T}$ : the mathematical angle for TC center viewing from the radar

$Z$ : $\quad$ radar reflectivity factor

\section{APPENDIX B}

\section{The Approximation of $\cos \alpha$}

From the triangle OTE in Fig. $1, \sin \alpha$ and $\cos \alpha$ can be written as follows: 


$$
\begin{aligned}
\frac{\sin \alpha}{R} & =\frac{\sin \psi}{R_{T}} \\
\sin \alpha & =\frac{R}{R_{T}} \sin \psi \\
\sin \alpha & =\sin \alpha_{\max } \sin \psi \\
\cos \alpha & =\left[1-\left(\sin \alpha_{\max } \sin \psi\right)^{2}\right]^{1 / 2} .
\end{aligned}
$$

Since $\cos \alpha$ cannot be explicitly expressed in terms of $\sin n \psi$ and $\cos n \psi$, an approximation form of $\cos \alpha$ is constructed as follows. Table B1 lists the corresponding $\cos \alpha$ with respect to $\psi$. It is clear that $\cos \alpha$ goes two cycles while $\psi$ varies from 0 to $2 \pi$ and can be approximated as a function of $\cos 2 \psi$. In addition, $\cos \alpha$ is bounded between $\cos \alpha_{\max }$ and 1. Therefore, $\cos \alpha$ has an amplitude of $\left(1-\cos \alpha_{\max }\right) / 2$ :

$$
\cos \alpha \approx \frac{1-\cos \alpha_{\max }}{2} \cos 2 \psi+\frac{1+\cos \alpha_{\max }}{2} .
$$

Figure B1 illustrates the approximation of $\cos \alpha$ in (B5) and its analytical expression in (5) at three different $\alpha_{\max }: 30^{\circ}, 60^{\circ}$, and $80^{\circ}$. The differences vary throughout the $0-2 \pi$ interval for a given $\alpha_{\max }$. It clearly indicates that the extreme errors of this approximation increase with ascending $\alpha_{\max }$ as illustrated in Table B2. The errors are less than $1 \%$ when $\alpha_{\max }$ is less than $45^{\circ}$. The errors exceed $10 \%$ when $\alpha_{\max }$ is beyond $75^{\circ}$. Therefore, the approximation becomes questionable only when $\alpha_{\max }$ is greater than $75^{\circ}$.

TABLE B1. The relationship among $\psi, \alpha$, and $\cos \alpha$.

\begin{tabular}{ccc}
\hline \hline$\psi$ & $\alpha$ & $\cos \alpha$ \\
\hline 0 & 0 & 1 \\
$\pi / 2$ & $\alpha_{\max }$ & $\cos \alpha_{\max }$ \\
$\pi$ & 0 & 1 \\
$3 \pi / 2$ & $-\alpha_{\max }$ & $\cos \alpha_{\max }$ \\
$2 \pi$ & 0 & 1 \\
\hline
\end{tabular}

TABLE B2. The maximum and minimum differences between $\cos \alpha$ and its approximation at different $\alpha_{\max }$.10-t202

\begin{tabular}{rcc}
\hline \hline$\alpha_{\max }\left({ }^{\circ}\right)$ & Max dif & Min dif \\
\hline 5 & $5.96046 \times 10^{-8}$ & $-1.78814 \times 10^{-6}$ \\
10 & 0.0 & $-2.90871 \times 10^{-5}$ \\
15 & 0.0 & $-1.47581 \times 10^{-4}$ \\
20 & 0.0 & $-4.68612 \times 10^{-4}$ \\
25 & 0.0 & $-1.15108 \times 10^{-3}$ \\
30 & 0.0 & $-2.40469 \times 10^{-3}$ \\
35 & 0.0 & $-4.49365 \times 10^{-3}$ \\
40 & 0.0 & $-7.74080 \times 10^{-3}$ \\
45 & 0.0 & $-1.25309 \times 10^{-2}$ \\
50 & 0.0 & $-1.94002 \times 10^{-2}$ \\
55 & 0.0 & $-2.88888 \times 10^{-2}$ \\
60 & 0.0 & $-4.16355 \times 10^{-2}$ \\
65 & 0.0 & $-5.85824 \times 10^{-2}$ \\
70 & 0.0 & $-8.05405 \times 10^{-2}$ \\
75 & 0.0 & -0.109077 \\
80 & 0.0 & -0.145381 \\
85 & 0.0 & -0.191243 \\
\hline
\end{tabular}
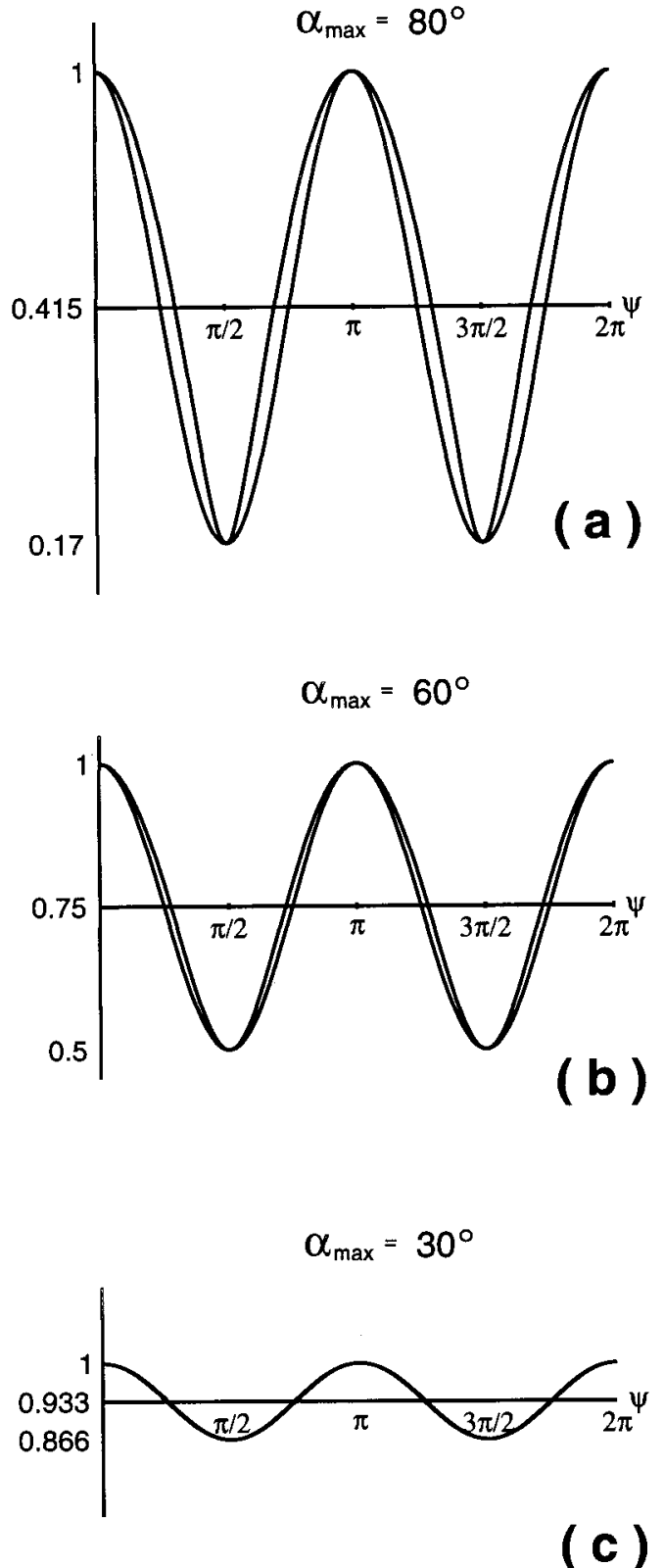

FIG. B1. The distribution of $\cos \alpha$ and its approximations (B5) as a function of $\psi$.

\section{REFERENCES}

Anthes, R. A., 1982: Tropical Cyclones-Their Evolution, Structure and Effects. Meteor. Monogr., No. 41, Amer. Meteor. Soc., 208 pp.

Baynton, H. W., 1979: The case for Doppler radars along our hurricane affected coasts. Bull. Amer. Meteor. Soc., 60, 1014-1023.

Brown, R. A., and V. T. Wood, 1991: On the interpretation of singleDoppler velocity patterns within severe thunderstorms. Wea. Forecasting, 6, 32-48.

, L. R. Lemon, and D. W. Burgess, 1978: Tornado detection by pulsed Doppler radar. Mon. Wea. Rev., 106, 29-38.

Browning, K. A., and R. Wexler, 1968: The determination of kine- 
matic properties of a wind field using Doppler radar. J. Appl. Meteor., 7, 105-113.

Caton, P. G. F., 1963: The measurement of wind and convergence by Doppler radar. Proc. 10th Conf. on Radar Meteorology, Washington, DC, Amer. Meteor. Soc., 290-296.

Donaldson, R. J., Jr., 1970: Vortex signature recognition by a Doppler radar. J. Appl. Meteor., 9, 661-670.

Griffin, J. S., R. W. Burpee, F. D. Marks Jr., and J. L. Franklin, 1992: Real-time airborne analysis of aircraft data supporting operational hurricane forecasting. Wea. Forecasting, 7, 480-490.

Hawkins, H. F., and D. T. Rubsam, 1968: Hurricane Hilda, 1964. Part II: Structure and budgets of the hurricane on October 1, 1964. Mon. Wea. Rev., 96, 617-636.

Jorgensen, D. P., 1984a: Mesoscale and convective-scale characteristics of mature hurricanes. Part I: General observations by research aircraft. J. Atmos. Sci., 41, 1268-1285.

_ 1984b: Mesoscale and convective-scale characteristics of mature hurricanes. Part II: Inner core structure of Hurricane Allen (1980). J. Atmos. Sci., 41, 1287-1131.

Jou, B. J.-D., P. L. Chang, and C.-S. Lee, 1997: Dual-Doppler analysis of a landfall typhoon rainband. Preprints, $22 d$ Conf. on Hurricanes and Tropical Meteorology, Fort Collins, CO, Amer. Meteor. Soc., 677-678.

LaSeur, N. E., and H. F. Hawkins, 1963: An analysis of Hurricane Cleo (1958) based on data from research reconnaissance aircraft. Mon. Wea. Rev., 91, 694-709.

Lee, W.-C., F. D. Marks, and R. E. Carbone, 1994: Velocity Track Display-A technique to extract real-time tropical cyclone circulations using a single airborne Doppler radar. J. Atmos. Oceanic Technol., 11, 337-356.

Lemon, L. R., D. W. Burgess, and R. A. Brown, 1978: Tornadic storm airflow and morphology derived from single Doppler radar measurements. Mon. Wea. Rev., 106, 48-61.

Lhermitte, R. M., and D. Atlas, 1962: Precipitation motion by pulse Doppler radar. Proc. Ninth Weather Radar Conf., Kansas City, MO, Amer. Meteor. Soc., 218-223.
Marks, F. D., and R. A. Houze, 1984: Airborne Doppler radar observations in Hurricane Debby. Bull. Amer. Meteor. Soc., 65, $569-582$.

— , and - 1987: Inner core structure of Hurricane Alicia from Doppler radar observations. J. Atmos. Sci., 44, 1296-1317.

- - - , and J. F. Gamache, 1992: Dual-aircraft investigation of the inner core of Hurricane Norbert. Part I: Kinematic structure. J. Atmos. Sci., 49, 919-942.

Matejka, T., and R. C. Srivastava, 1991: An improved version of the extended velocity-azimuth display technique for analyzing single-Doppler radar data. J. Atmos. Oceanic Technol., 8, 453-466.

Metcalf, J. I. and K. M. Glover, 1990: A history of weather radar research in the U.S. Air Force. Radar in Meteorology, D. Atlas, Ed., Amer. Meteor. Soc., 32-43.

Mohr, C. G., L. J. Miller, R. L. Vaughn, and H. W. Frank, 1986: Merger of mesoscale dataset into a common Cartesian format for efficient and systematic analysis. J. Atmos. Oceanic Technol., 3, 143-161.

Rankine, W. J. M., 1901: Motions of fluids. A Manual of Applied Mechanics, 16th ed., Charles Griff and Company, 574-578.

Roux, F., and N. Viltard, 1995: Structure and evolution of Hurricane Claudette on 7 September 1991 from airborne Doppler radar observations. Part I: Kinematics. Mon. Wea. Rev., 123, 26112639.

_, and F. D. Marks Jr., 1996: Extended Velocity Track Display (EVTD): An improved processing method for Doppler radar observations of tropical cyclones. J. Atmos. Oceanic Technol., 13, 875-899.

Shea, D. J., and W. M. Gray, 1973: The hurricane's inner core region. Part I: Symmetric and asymmetric structure. J. Atmos. Sci., 30, $1544-1564$

Stewart, S. R., and S. W. Lyons, 1996: A WSR-88D radar view of Tropical Cyclone Ed. Wea. Forecasting, 11, 115-135.

Wood, V. T., and R. A. Brown, 1992: Effects of radar proximity on single-Doppler velocity signatures of axisymmetric rotation and divergence. Mon. Wea. Rev., 120, 2798-2807. 